\title{
New metric properties for prox-regular sets.
}

\author{
S. Adly • F. Nacry • L. Thibault
}

Received: date / Accepted: date

\begin{abstract}
In this paper, we present diverse new metric properties that proxregular sets shared with convex ones. At the heart of our work lie the LegendreFenchel transform and complements of balls. First, we show that a connected prox-regular set is completely determined by the Legendre-Fenchel transform of a suitable perturbation of its indicator function. Then, we prove that such a function is also the right tool to extend, to the context of prox-regular sets, the famous connection between the distance function and the support function of a convex set. On the other hand, given a prox-regular set, we examine the intersection of complements of open balls containing the set. We establish that the distance of a point to a prox-regular set is the maximum of the distances of the point from boundaries of all such complements separating the set and the point. This is in the line of the known result expressing the distance from a convex set in terms of separating hyperplanes. To the best of our knowledge, these results are new in the literature and show that the class of prox-regular sets have good properties known in convex analysis.
\end{abstract}

Mathematics Subject Classification (2010) 49J52 · 49J53

Samir Adly

Laboratoire XLIM, Université de Limoges, 123, Avenue Albert Thomas, 87060 Limoges CEDEX, France. E-mail: samir.adly@unilim.fr

Florent Nacry

Laboratoire de Mathématiques et Physique, Université de Perpignan Via Domitia, 52 Avenue Paul Alduy, 66860 Perpignan. E-mail: florent.nacry@univ-perp.fr

Lionel Thibault

Université de Montpellier, Institut Montpelliérain Alexander Grothendieck 34095 Montpellier CEDEX 5 France. E-mail: lionel.thibault@umontpellier.fr 


\section{Introduction}

Context and motivations of the paper. The concept of prox-regularity finds its roots in the fundamental work [17] by H. Federer under the name positively reached sets. Federer by introducing the notion of curvature measure unified the studies of volumes of enlargements of sets in differential and convex geometry. This was a remarkable extension of Steiner polynomial formula, known for convex sets or $C^{2}$ submanifolds, to positively reached sets. The notion of prox-regular sets was introduced in the literature under different names over the years and through different but equivalent definitions. For example A. Canino ([12]) called it $p$-convexity, the name $\mathcal{O}(2)$-convex sets is due to A. Shapiro ([32]), J.-P. Vial ([35]) in 1983 introduced and studied the concept of weak convexity. Recently, F.H. Clarke, R.J. Stern and P.R. Wolenski ([15]) introduced the notion of proximally smooth sets, defined as closed sets $S$ in a Hilbert space $X$ such that the distance function $d_{S}$ is continuously differentiable on the open tube $\operatorname{Tube}_{r}(S):=\left\{x \in X: 0<d_{S}(x)<r\right\}$, for some $r>0$. The name prox-regularity was coined by R.A. Poliquin and R.T. Rockafellar ([29]) for functions, and prox-regular sets were studied by R.A. Poliquin, R.T. Rockafellar and L. Thibault ([30]) as sets whose indicator functions are prox-regular. It emerges from these various articles that the prox-regular and convex sets share many important properties such as the metric properties of Lipschitz continuity of the projection and the differentiability of the distance function on an appropriate tube. If the prox-regularity and the convexity enjoy for example these two fundamental properties, unfortunately there exist others, like intersection and inverse image by a linear mapping, which are not preserved for prox-regular sets. We refer the reader to [2] for counterexamples and sufficient conditions for such properties and others. Despite the absence of a good behavior of the prox-regularity with respect to set operations, we will see in the present paper that prox-regular sets share diverse new metric properties with convexity. This gives quite remarkable writings of the distance function from a prox-regular set.

Contributions of the paper. Let $S$ be a subset of a Hilbert space $X$ endowed with an inner product $\langle\cdot, \cdot\rangle$ and its associated norm $\|\cdot\|$. The usual support function $\sigma(\cdot, S)$ of $S$ is defined by $\sigma\left(x^{*}, S\right):=\sup _{x \in S}\left\langle x^{*}, x\right\rangle$. In Convex

Analysis, the concept of support functions for closed convex sets is known to be one of the most fundamental notions. It is often used to translate geometric Hahn-Banach separation theorems and in particular it characterizes a closed convex set $C$ through the following equivalence property: $x \in C$ if and only if $\left\langle x^{\star}, x\right\rangle \leq \sigma\left(x^{\star}, C\right)$ for all $x^{\star} \in X$. Recalling that the Legendre-Fenchel conjugate $f^{*}: X \rightarrow \mathbb{R} \cup\{-\infty,+\infty\}$ of a function $f: X \rightarrow \mathbb{R} \cup\{-\infty,+\infty\}$ is given by $f^{*}\left(x^{\star}\right):=\sup _{x \in X}\left(\left\langle x^{\star}, x\right\rangle-f(x)\right)$, it is clear and well-known that the support function $\sigma(\cdot, S)$ of the set $S$ is the Legendre-Fenchel conjugate of the indicator function $\psi_{S}$ of $S$ defined by $\psi_{S}(x)=0$ if $x \in S$ and $\psi_{S}(x)=+\infty$ if $x \in X \backslash S$. So, in Convex Analysis support functions are at the heart of many results related to duality theory, normal cones, barrier cones etc. (see, 
e.g., [4]). For a closed set $S$ of the Hilbert space $X$, E. Asplund ([5]) uses in an original way the Legendre-Fenchel transform of the function $\psi_{S}+\frac{1}{2}\|\cdot\|^{2}$ to characterize the convexity of $S$ via the single-valuedness and the continuity of the metric projection onto $S$. As we already mentioned in [1], Asplund's paper [5] seems to be the first one revealing the great interest of this function

$$
x^{\star} \mapsto\left(\psi_{S}+\frac{1}{2}\|\cdot\|^{2}\right)^{*}\left(x^{\star}\right)=\sup _{x \in S}\left(\left\langle x^{\star}, x\right\rangle-\frac{1}{2}\|x\|^{2}\right)
$$

in the study of the behavior of closed sets of Hilbert spaces. This function is strongly involved in approximation theory as highlighted by J.-B. HiriartUrruty $([22,23])$ who called it the Asplund function of $S$ and employed the notation $\varphi_{S}$. For other works using the function $\varphi_{S}$ we refer, for example, to $[8,33]$ and the references therein.

When the closed set $S$ of the Hilbert space $X$ is $r$-prox-regular for some $r>0$ (see Definition 1), it will be shown that the variant function $\varphi_{S, r}$ on $X$, defined by

$$
\varphi_{S, r}\left(x^{\star}\right):=\left(\psi_{S}+\frac{1}{2 r}\|\cdot\|^{2}\right)^{*}\left(x^{\star}\right)=\sup _{x \in S}\left(\left\langle x^{\star}, x\right\rangle-\frac{1}{2 r}\|x\|^{2}\right),
$$

is the right tool to extend the following fundamental results known for convex sets in convex analysis to the variational analysis of prox-regular sets:

$\left(\pi_{1}\right)$ A closed convex set is completely determined by its support function, in the sense that for two closed convex sets $C_{1}, C_{2}$

$$
C_{1}=C_{2} \Leftrightarrow \sigma\left(\cdot, C_{1}\right)=\sigma\left(\cdot, C_{2}\right) ;
$$

$\left(\pi_{2}\right)$ The analytic formulation of the distance from a convex set $C$ in terms of its support function (see, e.g., [20, Theorem 6.23])

$$
d_{C}(x)=\left\langle x^{\star}, x\right\rangle-\sigma\left(x^{\star}, C\right) \text { for some } x^{*} \in \mathbb{S}:=\{u \in X:\|u\|=1\} ;
$$

$\left(\pi_{3}\right)$ The duality property for a closed convex set $C$

$$
d_{C}(x)=\max _{x^{\star} \in \mathbb{S}} \inf _{y \in C}\left\langle x^{\star}, x-y\right\rangle=\inf _{y \in C} \max _{x^{\star} \in \mathbb{S}}\left\langle x^{\star}, x-y\right\rangle ;
$$

$\left(\pi_{4}\right)$ The formula for the distance from a closed convex set $C$ in terms of supporting hyperplanes: the distance $d_{C}(x)$ coincides with the maximum of distances $d_{H}(x)$ taken over all hyperplanes $H$ separating $C$ and $x \notin C$, and this maximum is attained for one and only one hyperplane.

The extensions of the above properties $\left(\pi_{1}\right)-\left(\pi_{4}\right)$ to the prox-regular setting are respectively developed in Theorem 4-Corollary 1 , Theorem 6 , Proposition 5 and Theorem 7.

Organization of the paper. After some preliminaries in Section 2, we investigate in Section 3 the great role played by the function $\varphi_{S, r}$, defined in (1), in the context of $r$-prox-regular sets. The last section of the present work is devoted to the distance function associated to a prox-regular set. 


\section{Notation and preliminaries}

As usual, $\mathbb{N}$ denotes the set of integers starting from 1 and $\overline{\mathbb{R}}:=\mathbb{R} \cup\{-\infty,+\infty\}$ is the extended real-line. Given an extended real $r \in \overline{\mathbb{R}}$, we will denote by $r^{+}$ its positive part, that is, $r^{+}:=\max \{r, 0\}$.

Throughout the paper, $X$ stands for a (real) Hilbert space not reduced to the trivial space $\{0\}$ endowed with the inner product $\langle\cdot, \cdot\rangle$ and its associated norm $\|\cdot\|:=\sqrt{\langle\cdot, \cdot\rangle}$. The open (resp. closed) ball and the sphere of $X$ centered at $x \in X$ with radius $\rho>0$ are denoted by $B(x, \rho)(\operatorname{resp} . B[x, \rho])$ and $S(x, \rho)$. In the particular case of the closed unit ball and the unit sphere of $X$, we use the following notation

$$
\mathbb{B}:=B\left[0_{X}, 1\right] \text { and } \mathbb{S}:=S\left(0_{X}, 1\right) .
$$

2.1 Distance function and associated sets

Now, consider any nonempty closed subset $S$ of $X$. The distance function $d_{S}$ from $S$ is defined as

$$
d_{S}(x):=: d(x, S)=\inf _{y \in S}\|x-y\| \quad \text { for all } x \in X .
$$

Given any nonzero vector $x^{\star}$ in the Hilbert space $X$ and any $\alpha \in \mathbb{R}$, it is known (see, e.g., [20]) that the distance function of $\bar{x} \in X$ from the affine hyperplane $H_{=}\left(x^{\star}, \alpha\right):=\left\{x \in X:\left\langle x^{\star}, x\right\rangle=\alpha\right\}=:\left\{x^{\star}=\alpha\right\}$ (resp. from the closed affine half-space $\left.H_{\leq}\left(x^{\star}, \alpha\right):=\left\{x \in X:\left\langle x^{\star}, x\right\rangle \leq \alpha\right\}=:\left\{x^{\star} \leq \alpha\right\}\right)$ is given by

$$
d\left(\bar{x}, H_{=}\left(x^{\star}, \alpha\right)\right)=\left\|x^{\star}\right\|^{-1}\left|\left\langle x^{\star}, \bar{x}\right\rangle-\alpha\right|
$$

(resp.

$$
\left.d\left(x, H_{\leq}\left(x^{\star}, \alpha\right)\right)=\left\|x^{\star}\right\|^{-1}\left(\left\langle x^{\star}, \bar{x}\right\rangle-\alpha\right)^{+}\right) .
$$

The multimapping $\operatorname{Proj}_{S}: X \rightrightarrows X$ of nearest points in $S$ is defined by

$$
\operatorname{Proj}_{S}(x):=: \operatorname{Proj}(S, x):=\left\{y \in S:\|x-y\|=d_{S}(x)\right\} \quad \text { for all } x \in X .
$$

Whenever the latter set is reduced to a singleton for some $\bar{x} \in X$, that is, $\operatorname{Proj}_{S}(\bar{x})=\{\bar{y}\}$, the vector $\bar{y} \in S$ will be denoted by $\operatorname{proj}_{S}(\bar{x})$ or $P_{S}(\bar{x})$. We say that $S$ is proximinal provided that $\operatorname{Proj}_{S}(x) \neq \emptyset$ for every $x \in X$. It is known and not difficult to check that every nonempty weakly closed subset of $X$ is proximinal.

Given an extended real $r \in] 0,+\infty$ ], we define the (open) $r$-tube of $S$ as the set $\operatorname{Tube}_{r}(S):=U_{r}(S) \backslash S$ where $U_{r}(S)$ is the (open) $r$-enlargement of $S$

$$
U_{r}(S):=\left\{x \in X: d_{S}(x)<r\right\}
$$

and we also introduce the (closed) $r$-exterior of $S$ and the set of points at exact $r$-distance to $S$

$$
\operatorname{Exte}_{r}(S):=\left\{x \in X: d_{S}(x) \geq r\right\} \quad \text { and } \quad D_{r}(S):=\left\{x \in X: d_{S}(x)=r\right\} .
$$

If $r=+\infty$, such sets become $U_{\infty}(S):=X$ and $\operatorname{Ext}_{\infty}(S)=\emptyset=D_{\infty}(S)$. 


\subsection{Proximal normals}

The proximal normal cone of a closed subset $S \subset X$ at $x \in S$, denoted by $\mathrm{N}^{P}(S ; x)$, is defined as

$$
\mathrm{N}^{P}(S ; x):=\left\{v \in X: \exists r>0, x \in \operatorname{Proj}_{S}(x+r v)\right\}
$$

It is not difficult to see that for every $v \in X$, the inclusion $v \in \mathrm{N}^{P}(S ; x)$ holds if and only if there is a real $r>0$ satisfying

$$
\left\langle v, x^{\prime}-x\right\rangle \leq \frac{1}{2 r}\left\|x^{\prime}-x\right\|^{2} \quad \text { for all } x^{\prime} \in S
$$

in such a case we will say that $v$ is a proximal normal to $S$ at $x$ with constant $r>0$. If $x \notin S$, we set by convention $\mathrm{N}^{P}(S ; x):=\emptyset$. According to the definition, we notice that, for any $v \in X$ such that $\operatorname{Proj}_{S}(v) \neq \emptyset$, we have

$$
v-w \in \mathrm{N}^{P}(S ; w) \quad \text { for all } w \in \operatorname{Proj}_{S}(v) .
$$

It is worth pointing out that the above concept of normal cone is local, in the sense that for any neighborhood $V$ in $X$ of $x \in S$

$$
\mathrm{N}^{P}(S \cap V ; x)=\mathrm{N}^{P}(S ; x) .
$$

If $S$ is convex, it is known (and easily seen) that the proximal normal cone $\mathrm{N}^{P}(S ; x)$ coincides with the normal cone in the sense of convex analysis, i.e.,

$$
\mathrm{N}^{P}(S ; x)=\{v \in X:\langle v, y-x\rangle \leq 0, \forall y \in S\}
$$

Given a function $f: U \rightarrow \overline{\mathbb{R}}$ defined on a nonempty open subset $U \subset X$, one says that a vector $\zeta \in X$ is a proximal subgradient of $f$ at a point $x \in U$ with $f(x)$ finite, provided that $(\zeta,-1) \in \mathrm{N}^{P}$ (epi $\left.f ;(x, f(x))\right)$, where epi $f$ is the epigraph of $f$, that is the subset of $\mathcal{H} \times \mathbb{R}$ (endowed with its usual Hilbert product structure) defined by epi $f:=\{(u, r) \in U \times \mathbb{R}: u \in U, f(u) \leq r\}$. It is well-known (and not difficult to check) that the latter inclusion holds if and only if there are a real $\sigma \geq 0$ and a real $\eta>0$ with $B(x, \eta) \subset U$ such that

$$
\langle\zeta, y-x\rangle \leq f(y)-f(x)+\sigma\|y-x\|^{2} \quad \text { for all } y \in B(x, \eta)
$$

The set $\partial_{P} f(x)$ of all proximal subgradients of $f$ at $x$ is the proximal subdifferential of $f$ at $x$. In the same spirit as (5), whenever the set $U$ and the fonction $f$ are convex, the proximal subdifferential $\partial_{P} f(x)$ coincide with the subdifferential of convex analysis $\partial f(x)$, that is,

$$
\partial_{P} f(x)=\{\zeta \in X:\langle\zeta, y-x\rangle \leq f(y)-f(x), \forall y \in U\}=: \partial f(x) .
$$


2.3 Prox-regularity

This succinct paragraph is devoted to the needed basic facts on prox-regularity which is at the heart of the present paper. For more details on that topic, in both theoretical and concrete approaches, we refer for instance to [30], the survey [16] and the book [34] along with the references therein.

Definition 1 Let $S$ be a nonempty closed subset of $X$ and $r \in] 0,+\infty]$. One says that $S$ is $r$-prox-regular whenever, for all $x \in S$, for all $v \in \mathrm{N}^{P}(S ; x) \cap \mathbb{B}$ and for every real $t \in] 0, r]$, one has $x \in \operatorname{Proj}_{S}(x+t v)$.

Given a closed subset $S \subset X, x \in S$ and $v \in \mathrm{N}^{P}(S ; x)$ with $\|v\|=1$, it is routine to check that for every real $t>0$ one has

$$
x \in \operatorname{Proj}_{S}(x+t v) \Leftrightarrow S \cap B(x+t v, t)=\emptyset .
$$

In such a case, one says that the unit normal proximal vector $v$ to $S$ at $x$ is realized by the t-ball $B(x+t v, t)$.

The following theorem provides some useful characterizations and properties of uniform prox-regular sets for which we refer to $[30,16,34]$.

Theorem 1 Let $S$ be a nonempty closed subset of $X$ and let $r \in] 0,+\infty]$. The following assertions are equivalent.

(a) The set $S$ is r-prox-regular.

(b) For all $x, x^{\prime} \in S$, for all $v \in \mathrm{N}^{P}(S ; x)$, one has

$$
\left\langle v, x^{\prime}-x\right\rangle \leq \frac{1}{2 r}\|v\|\left\|x-x^{\prime}\right\|^{2} .
$$

(c) On $U_{r}(S)$ the mapping $P_{S}(\cdot)$ is well-defined, and for every real $\left.s \in\right] 0, r[$, for all $x, x^{\prime} \in U_{s}(S)$,

$$
\left\|P_{S}(x)-P_{S}\left(x^{\prime}\right)\right\| \leq \frac{1}{1-(s / r)}\left\|x-x^{\prime}\right\| .
$$

(d) For all $x \in \operatorname{Tube}_{r}(S)$, the vector $P_{S}(x)$ is well defined and one has

$$
P_{S}(x)=P_{S}\left(P_{S}(x)+t \frac{x-P_{S}(x)}{\left\|x-P_{S}(x)\right\|}\right) \quad \text { for all } t \in[0, r[.
$$

(e) The function $d_{S}^{2}(\cdot)$ is differentiable on $U_{r}(S)$ with a locally Lipschitz derivative and

$$
\nabla d_{S}^{2}(x)=2\left(x-P_{S}(x)\right) \quad \text { for all } x \in U_{r}(S) .
$$

Remark 1 It should be noted that for an $r$-prox-regular subset of $X$ with $r \in] 0,+\infty[$, we have

$$
P_{S}(x) \in \operatorname{Proj}_{S}\left(P_{S}(x)+r \frac{x-P_{S}(x)}{d_{S}(x)}\right) \text { for all } x \in \operatorname{Tube}_{r}(S) .
$$




\section{Legendre-Fenchel transform}

As mentioned in the introduction, E. Asplund seems to be the first to depict, for a (non necessarily convex) set $S$ in a Hilbert space, the importance of the Legendre-Fenchel conjugate of the function $\psi_{S}+\frac{1}{2}\|\cdot\|^{2}$, that is, the function

$$
x^{\star} \mapsto \sup _{y \in X}\left(\left\langle x^{\star}, y\right\rangle-\left(\psi_{S}(y)+\frac{1}{2}\|y\|^{2}\right)\right)=\sup _{y \in S}\left(\left\langle x^{\star}, y\right\rangle-\frac{1}{2}\|y\|^{2}\right) .
$$

This function is denoted by $\varphi_{S}$ in [8], and Asplund called it the indefinite integral of the metric projection of $S$, a term due to the inclusion

$$
p \in \partial \varphi_{S}(x) \quad \text { whenever } p \in \operatorname{Proj}_{S}(x) \neq \emptyset .
$$

Computing the biconjugate of $\psi_{S}+\frac{1}{2}\|\cdot\|^{2}$, that is, the (Legendre-Fenchel) conjugate of $\varphi_{S}$ and applying the theory of convex analysis, Asplund established in [5] with a very elegant and short way the result in Theorem 2 on Chebyshev set. Recall that a nonempty closed set $S$ in $X$ is a Chebyshev set if any point in $X$ admits one and only one nearest point in $S$.

Theorem 2 If the metric projection onto a Chebyshev set in a Hilbert space is norm-to-weak continuous, then the set is convex.

A decade later, H. Berens ([8]) gave a full description of subgradients of the convex function $\varphi_{S}$ thanks to the theory of maximal monotone operators. For any nonempty closed set $S \subset X$, Berens established that

$$
\partial \varphi_{S}(x)=\bigcap_{\varepsilon>0} \overline{\operatorname{co}}\left(\operatorname{Proj}_{S, \varepsilon}(x)\right) \quad \text { for all } x \in X,
$$

where as usual $\operatorname{Proj}_{S, \varepsilon}(x):=\left\{s \in S:\|x-s\|<d_{S}(x)+\varepsilon\right\} \neq \emptyset$. Taking into account (8) or equivalently the inclusion

$$
\overline{\mathrm{co}}\left(\operatorname{Proj}_{S}(x)\right) \subset \partial \varphi_{S}(x) \text { for all } x \in X
$$

(keep in mind that $\partial \varphi_{S}(\cdot)$ has closed convex values), H. Berens asked the following question: if $\operatorname{dim} X=+\infty$, is it possible to find a proximinal set $S \subset X$ and $\bar{x} \in X$ such that

$$
\overline{\mathrm{co}}\left(\operatorname{Proj}_{S}(\bar{x})\right) \neq \partial \varphi_{S}(\bar{x}) ?
$$

The answer is positive for any infinite dimensional Hilbert space and it was given by G. Godini in [19].

The function $\varphi_{S}(\cdot)$ also appears under the name Asplund function in the two surveys devoted to challenging open problems in approximation theory, namely convexity of Chebyshev sets ([22]) and farthest points conjecture ([23]). In [22], the function $\varphi_{S}$ allows to show that $d_{S}^{2}$ is differentiable on $\mathbb{R}^{n}$ if and only if $S$ is convex. We also mention that in [23], Hiriart-Urruty developed among other things a new technique to establish (9) only with tools coming from convex analysis (see also the survey [33]). 
Given any set $S \subset X$ and any extended real $r>0$, it will be relevant to introduce the following variant (which could be called Asplund function of constant $r) \varphi_{S, r}: X \rightarrow \overline{\mathbb{R}}$ of the function $\varphi_{S}$ defined by

$$
\varphi_{S, r}\left(x^{\star}\right):=\sup _{y \in S}\left(\left\langle x^{\star}, y\right\rangle-\frac{\|y\|^{2}}{2 r}\right) \quad \text { for all } x^{\star} \in X .
$$

We immediately observe that $\varphi_{S, r} \equiv-\infty$ when $S=\emptyset$, hence there will be no loss of generality to assume that $S \neq \emptyset$. Whenever $r=+\infty$ (resp. $r=1$ ), it is of interest to note that the function $\varphi_{S, \infty}$ (resp. $\left.\varphi_{S, 1}\right)$ is nothing but the usual support (resp. Asplund) function of $S$, that is,

$$
\varphi_{S, \infty}\left(x^{\star}\right):=\sup _{y \in S}\left\langle x^{\star}, y\right\rangle=: \sigma\left(x^{\star}, S\right) \quad \text { for all } x^{\star} \in X
$$

(resp.

$$
\left.\varphi_{S, 1}\left(x^{\star}\right):=\sup _{y \in S}\left(\left\langle x^{\star}, y\right\rangle-\frac{\|y\|^{2}}{2}\right)=: \varphi_{S}\left(x^{\star}\right) \quad \text { for all } x^{\star} \in X\right) .
$$

It is worth pointing out that $\varphi_{S, r}$ is, as $\varphi_{S}$, the (classical) Legendre-Fenchel conjugate of a function, namely

$$
\left(\psi_{S}+\frac{1}{2 r}\|\cdot\|^{2}\right)^{\star}=\varphi_{S, r} .
$$

The equality (10) (for which the important particular case $r=1$ has been stated in (7)) leaded us in [1] to set for a prescribed function $f: X \rightarrow \overline{\mathbb{R}}$

$$
f^{\star, r}\left(x^{\star}\right):=\left(f+\frac{1}{2 r}\|\cdot\|^{2}\right)^{\star}\left(x^{\star}\right) \quad \text { for all } x^{\star} \in X .
$$

Accordingly, we can rewrite (10) as

$$
\left(\psi_{S}\right)^{\star, r}=\varphi_{S, r}
$$

so (7) becomes $\left(\psi_{S}\right)^{\star, 1}=\varphi_{S, 1}$. From the very definition of $\varphi_{S, r}$ we also see that the inequality $\varphi_{S, r}\left(x^{\star}\right) \leq \alpha$ is equivalent to the inclusion $S \subset Q_{x^{\star}, r, \alpha}$, where the set in the right-hand side is defined (see [1]) as

$$
Q_{x^{\star}, r, \alpha}:=\left\{x \in X:\left\langle x^{\star}, x\right\rangle-\frac{\|x\|^{2}}{2 r} \leq \alpha\right\} .
$$

It is worth pointing out that for every $x^{\star} \in X \backslash\{0\}$ and every real $\alpha$, the set $Q_{x^{\star}, \infty, \alpha}$ is nothing but the (closed) half-space $H_{\leq}\left(x^{\star}, \alpha\right)$. Both above properties among others are stated in the following proposition. 
Proposition 1 Let $S$ be a nonempty subset of $X, r \in] 0,+\infty]$ be an extended real. The following hold.

(a) If $r<+\infty$, one has

$$
\varphi_{\mathrm{cl} S, r}\left(x^{\star}\right)=\varphi_{S, r}\left(x^{\star}\right)=\frac{r}{2}\left\|x^{\star}\right\|^{2}-\frac{1}{2 r} d_{S}^{2}\left(r x^{\star}\right) \quad \text { for all } x^{\star} \in X .
$$

In particular $\varphi_{S, r}$ is real-valued whenever $r<+\infty$.

(b) One has $\varphi_{S, r}(0)=-\frac{1}{2 r} d_{S}^{2}(0)$.

(c) For every real $\lambda \geq 0$, one has

$$
\varphi_{S, r}\left(\lambda x^{\star}\right)=\lambda \varphi_{S, r \lambda}\left(x^{\star}\right) \quad \text { for all } x^{\star} \in X .
$$

In particular, if $r<+\infty$, then one has

$$
r \varphi_{S, r}\left(x^{\star}\right)=\varphi_{S, 1}\left(r x^{\star}\right) \quad \text { for all } x^{\star} \in X .
$$

(d) The function $\varphi_{S, r}(\cdot)$ is the r-conjugate of $\psi_{S}$, i.e.,

$$
\varphi_{S, r}\left(x^{\star}\right)=\left(\psi_{S}\right)^{\star, r}\left(x^{\star}\right)=\left(\psi_{S}+\frac{1}{2 r}\|\cdot\|\right)^{\star}\left(x^{\star}\right) \quad \text { for all } x^{\star} \in X .
$$

(e) The function $\varphi_{S, r}(\cdot)$ is convex and locally Lipschitz continuous on $X$.

(f) For every $x^{\star} \in X$ and every $\alpha \in \mathbb{R}$, one has

$$
S \subset Q_{x^{\star}, r, \alpha} \Leftrightarrow \varphi_{S, r}\left(x^{\star}\right) \leq \alpha .
$$

Proof The assertion $(a)$ follows from the computation valid for every $x^{\star} \in X$,

$$
\varphi_{S, r}\left(x^{\star}\right)=\frac{r}{2}\left(\left\|x^{\star}\right\|^{2}+\sup _{x \in S}-\left\|x^{\star}-\frac{x}{r}\right\|^{2}\right)=\frac{r}{2}\left\|x^{\star}\right\|^{2}-\frac{1}{2 r} d_{S}^{2}\left(r x^{\star}\right) .
$$

The assertions $(b),(c)$ and $(f)$ are direct consequences of the definition of $\varphi_{S, r}$. Further, $(d)$ has already been established above. To prove $(e)$, it suffices to observe on one hand that the local Lipschitz property directly derives from (a) and on the other hand the convexity of $\varphi_{S, r}$ follows from the convexity of each $f_{y}: X \rightarrow \mathbb{R}$ with $y \in S$ defined by

$$
f_{y}(x):=\left\langle x^{\star}, y\right\rangle-\frac{\|y\|^{2}}{2 r} \quad \text { for all } x \in X .
$$

The proof is complete.

Assertion $(f)$ in Proposition 1 can be seen as an extension of the classical fact that the inequality $\sigma(\cdot, S) \leq \alpha$ characterizes the closed half-spaces which contain $S$, that is, for every $x^{\star} \in X$ with $x^{\star} \neq 0$ and every $\alpha \in \mathbb{R}$,

$$
S \subset\left\{\left\langle x^{\star}, \cdot\right\rangle \leq \alpha\right\}=\mathcal{H}_{\leq}\left(x^{\star}, \alpha\right)=: Q_{x^{\star}, \infty, \alpha} \Leftrightarrow \varphi_{S, \infty}\left(x^{\star}\right):=\sigma\left(x^{\star}, S\right) \leq \alpha .
$$

On the other hand, assertion $(a)$ in Proposition 1 makes clear that we cannot expect in general explicit formulas for the function $\varphi_{S, r}$ for any type of subset $S \subset X$ since this requires to be able to compute the distance function $d_{S}(\cdot)$. 
Example 1 Let $x \in X$ and $r>0$ be a real.

(i) For each real $\rho>0$, we have

$$
\varphi_{\rho \mathbb{B}, r}(x)= \begin{cases}\frac{r\|x\|^{2}}{2} & \text { if } x \in \frac{\rho}{r} \mathbb{B}, \\ \rho\|x\|-\frac{\rho^{2}}{2 r} & \text { otherwise. }\end{cases}
$$

(ii) For each real $\rho>0$ we have with $S:=X \backslash B(0, \rho)$

$$
\varphi_{S, r}(x)= \begin{cases}\rho\|x\|-\frac{\rho^{2}}{2 r} & \text { if }\|x\| \leq \rho, \\ \frac{r}{2}\|x\|^{2} & \text { otherwise. }\end{cases}
$$

(iii) Let $u^{\star} \in X \backslash\{0\}$ and $\alpha \in \mathbb{R}$. According to (2) and (3) we have with $\mathcal{H}:=\mathcal{H}_{\leq}\left(u^{\star}, \alpha\right)$ and $H:=H_{=}\left(u^{\star}, \alpha\right)$

$$
\varphi_{\mathcal{H}, r}(x)=\frac{r}{2}\|x\|^{2}-\frac{1}{2 r\left\|u^{\star}\right\|^{2}}\left[\left(r\left\langle u^{\star}, x\right\rangle-\alpha\right)^{+}\right]^{2} .
$$

and

$$
\varphi_{H, r}(x)=\frac{r}{2}\|x\|^{2}-\frac{1}{2 r\left\|u^{\star}\right\|^{2}}\left[\left(r\left\langle u^{\star}, x\right\rangle-\alpha\right)\right]^{2} .
$$

Now, we develop certain properties of the (sub)differential of the function $\varphi_{S, r}$ with $S \subset X$ and $\left.\left.r \in\right] 0,+\infty\right]$. Let us say that the set $S$ is strongly ballcompact provided that the intersection of $S$ with every closed ball of $X$ is strongly compact. If $S \subset X$ is nonempty, closed and strongly ball-compact, it is known (see, e.g., [27, Proposition 2.2]) that

$$
\partial_{C}\left(\frac{1}{2} d_{S}^{2}\right)(x)=x-\overline{\mathrm{co}}\left(\operatorname{Proj}_{S}(x)\right) \text { for all } x \in X \backslash S,
$$

where $\partial_{C}$ denotes the Clarke subdifferential (see $[14,31,28,26,34]$ and the references therein). The next proposition provides various descriptions of the subdifferential $\partial \varphi_{S, r}\left(x^{*}\right)$ for $x^{*} \in X$. The description in the first assertion is in terms of the following particular function associated to a vector $x^{\star} \in X$ $q_{x^{\star}, r}: X \rightarrow \mathbb{R}$ defined by

$$
q_{x^{\star}, r}(x):=\left\langle x^{\star}, x\right\rangle-\frac{\|x\|^{2}}{2 r} \text { for all } x \in X .
$$

This function already played a fundamental role in our previous paper [1]. The function $\varphi_{S, r}(\cdot)$ can be rewritten as

$$
\varphi_{S, r}\left(x^{\star}\right)=\sup q_{x^{\star}, r}(S) \quad \text { for all } x^{\star} \in X .
$$

Notice also that the set $Q_{x^{\star}, r, \alpha}$ in (11) is just the $\alpha$-sublevel of this function $q_{x^{\star}, r}(\cdot)$, that is, $Q_{x^{\star}, r, \alpha}:=\left\{q_{x^{\star}, r} \leq \alpha\right\}$, and clearly $q_{x^{\star}, \infty}(\cdot)$ coincides with the linear functional $\left\langle x^{\star}, \cdot\right\rangle$. 
Proposition 2 Let $S$ be a nonempty closed subset of $X$ and $r \in] 0,+\infty[$, and let $U$ be an open subset of $X$. The following hold:

(a) One always has

$$
S \cap \partial \varphi_{S, r}\left(x^{\star}\right)=\left\{x \in S: \varphi_{S, r}\left(x^{\star}\right)=q_{x^{\star}, r}(x)\right\} \quad \text { for all } x^{\star} \in X,
$$

and the latter equality still holds for $r=+\infty$.

(b) The function $\varphi_{S, r}(\cdot)$ is Fréchet differentiable on $U$ if and only if $d_{S}^{2}$ is Fréchet differentiable on $U$.

(c) Let $\rho>0$ be an extended real. The set $S$ is $\rho$-prox-regular if and only if $\varphi_{S, r}(\cdot)$ is Fréchet differentiable on $U_{\rho}(S)$. In such a case, one has

$$
\nabla \varphi_{S, r}(x)=\nabla \varphi_{S}(r x)=\left\{\operatorname{proj}_{S}(r x)\right\} \quad \text { for all } x \in U_{\rho}(S) .
$$

(d) One has

$$
\partial \varphi_{S, r}(x)=\bigcap_{\varepsilon>0} \overline{\operatorname{co}}\left(\operatorname{Proj}_{S, \varepsilon}(r x)\right) \quad \text { for all } x \in X
$$

(e) If $S$ is strongly ball-compact, then

$$
\partial \varphi_{S, r}(x)=\overline{\mathrm{co}}\left(\operatorname{Proj}_{S}(r x)\right) \quad \text { for all } x \in X \backslash r^{-1} S .
$$

(f) If $\operatorname{dim} X=+\infty$, there exist a proximinal set $S \subset X$ and $x \in X$ such that

$$
\partial \varphi_{S, r}(x) \neq \overline{\mathrm{co}}\left(\operatorname{Proj}_{S}(r x)\right) .
$$

Proof (a) Fix any $x^{\star} \in X$. Let $\bar{x} \in S$. Assume first that $\varphi_{S, r}\left(x^{\star}\right)=q_{x^{\star}, r}(\bar{x})$. Pick any $x^{\prime} \in X$. Thanks to the inclusion $\bar{x} \in S$, we have

$$
\left\langle x^{\prime}, \bar{x}\right\rangle \leq \sup _{u \in S}\left(\left\langle x^{\prime}, u\right\rangle-\frac{\|u\|^{2}}{2 r}\right)+\frac{\|\bar{x}\|^{2}}{2 r}
$$

which is obviously equivalent to

$$
\left\langle\bar{x}, x^{\prime}-x^{\star}\right\rangle \leq \sup _{u \in S}\left(\left\langle x^{\prime}, u\right\rangle-\frac{\|u\|^{2}}{2 r}\right)-\left\langle x^{\star}, \bar{x}\right\rangle+\frac{\|\bar{x}\|^{2}}{2 r} .
$$

The latter inequality can be rewritten as

$$
\left\langle\bar{x}, x^{\prime}-x^{\star}\right\rangle \leq \varphi_{S, r}\left(x^{\prime}\right)-q_{x^{\star}, r}(\bar{x})=\varphi_{S, r}\left(x^{\prime}\right)-\varphi_{S, r}\left(x^{\star}\right)
$$

and this justifies the inclusion $\bar{x} \in S \cap \partial \varphi_{S, r}\left(x^{\star}\right)$. Conversely, assume that $\bar{x} \in S \cap \partial \varphi_{S, r}\left(x^{\star}\right)$. From the definition of subdifferential of convex analysis (see (6)) it is not difficult to observe that

$$
\varphi_{S, r}\left(x^{\star}\right) \leq\left\langle x^{\star}, \bar{x}\right\rangle-\left\langle\bar{x}, x^{\prime}\right\rangle+\varphi_{S, r}\left(x^{\prime}\right) \quad \text { for all } x^{\prime} \in X .
$$

According to Proposition 1, we then deduce

$$
\varphi_{S, r}\left(x^{\star}\right) \leq\left\langle x^{\star}, \bar{x}\right\rangle-\left\langle\bar{x}, x^{\prime}\right\rangle+\frac{r}{2}\left\|x^{\prime}\right\|^{2}-\frac{1}{2 r} d_{S}^{2}\left(r x^{\prime}\right) \quad \text { for all } x^{\prime} \in X .
$$


Applying the latter inequality with the vector $\frac{\bar{x}}{r}$ yields

$$
\varphi_{S, r}\left(x^{\star}\right) \leq\left\langle x^{\star}, \bar{x}\right\rangle-\left\langle\bar{x}, \frac{\bar{x}}{r}\right\rangle+\frac{r}{2}\left\|\frac{\bar{x}}{r}\right\|^{2},
$$

otherwise stated $\varphi_{S, r}\left(x^{\star}\right) \leq\left\langle x^{\star}, \bar{x}\right\rangle-\frac{\|\bar{x}\|^{2}}{2 r}=q_{x^{\star}, r}(\bar{x}) \leq \varphi_{S, r}\left(x^{\star}\right)$. The proof of (a) is then complete.

(b) It is a direct consequence of Proposition 1(a).

(c) It suffices to put together $(b)$ above, Theorem 1(e) and the equality (12)

$$
\varphi_{S, r}=r^{-1}\left(\varphi_{S} \circ r \operatorname{Id}_{X}\right) .
$$

(d) Combining Beren's equality (9) with (17) gives the desired result.

(e) It follows from the two equalities (13) and (17) mentioned above.

$(f)$ This can be obtained through the Godini's construction ([19]) which provides a proximinal subset $S \subset X$ and $x \in X$ such that

$$
\partial \varphi_{S}(x) \neq \overline{\mathrm{co}}\left(\operatorname{Proj}_{S}(x)\right) .
$$

The right hand-side of (16) is strongly connected to the following concept of support points.

Definition 2 ([1]) Let $S$ be a closed subset of $X$ and $r \in] 0,+\infty]$ be an extended real. One says that $u^{\star} \in X$ is an $r$-quadratic support functional of $S$ whenever there exists $\bar{x} \in S$ such that

$$
q_{u^{\star}, r}(\bar{x}):=\left\langle x^{\star}, \bar{x}\right\rangle-\frac{1}{2 r}\|\bar{x}\|^{2}=\sup _{x \in S}\left(\left\langle u^{\star}, x\right\rangle-\frac{1}{2 r}\|x\|^{2}\right)=: \varphi_{S, r}\left(u^{\star}\right) .
$$

In such a case, $u^{\star}$ is said to quadratically support $S$ at $\bar{x}$. One says that $\bar{x} \in S$ is an $r$-quadratic support point of $S$ whenever there exists $u^{\star} \in X$ with $u^{\star} \neq \frac{\bar{x}}{r}$ such that $u^{\star}$ supports $S$ at $\bar{x}$.

Before going further, let us introduce a notation. To a nonempty closed subset $S$ of $X$, we associate the multimapping $\Gamma_{S, r}(\cdot): X \backslash S \rightrightarrows X$ defined by

$$
\Gamma_{S, r}(x):=\left(\frac{1}{r}-\frac{1}{d_{S}(x)}\right) \operatorname{Proj}_{S}(x)+\frac{1}{d_{S}(x)} x \quad \text { for all } x \in X \backslash S .
$$

When $\operatorname{Proj}_{S}(\bar{x})$ is reduced to a singleton for some $\bar{x} \in X \backslash S$ (i.e., $\operatorname{proj}_{S}(\bar{x})$ is well defined), the set $\Gamma_{S, r}(\bar{x})$ is obviously a singleton. In such a case, the set $\Gamma_{S, r}(\bar{x})$ will be identified to its unique element $\left(\frac{1}{r}-\frac{1}{d_{S}(\bar{x})}\right) \operatorname{proj}_{S}(\bar{x})+\frac{1}{d_{S}(\bar{x})} \bar{x}$. If in addition the set $S$ is $r$-prox-regular for some real $r>0$, we derive from Remark 1 the inclusions

$$
P_{S}(x) \in \operatorname{Proj}_{S}\left(r \Gamma_{S, r}(x)\right) \quad \text { for all } x \in \operatorname{Tube}_{r}(S)
$$

and

$$
\Lambda_{r}(S):=r \Gamma_{S, r}\left(\operatorname{Tube}_{r}(S)\right) \subset D_{r}(S) .
$$

Now, we are in position to recall the following theorem from [1]. 
Theorem 3 Let $S$ be an $r$-prox-regular subset of $X$ with $r \in] 0,+\infty], x \in$ $\operatorname{Tube}_{r}(S)$. Then, one has with $x^{\star}:=\Gamma_{S, r}(x)=\left(\frac{1}{r}-\frac{1}{d_{S}(x)}\right) \operatorname{proj}_{S}(x)+\frac{1}{d_{S}(x)} x$ the following separation property for some $\alpha \in \mathbb{R}$

$$
S \subset\left\{\left\langle x^{\star}, \cdot\right\rangle-\frac{\|\cdot\|^{2}}{2 r}<\alpha\right\} \subset Q_{x^{\star}, r, \alpha}
$$

and

$$
q_{x^{\star}, r}(x):=\left\langle x^{\star}, x\right\rangle-\frac{\|x\|^{2}}{2 r}>\alpha \geq \sup q_{x^{\star}, r}(S)=: \varphi_{S, r}\left(x^{\star}\right) .
$$

Concerning the $\alpha$-sublevel set $Q_{x^{\star}, r, \alpha}$ of $q_{x^{\star}, r}$ (see (11) and (14)), we also recall the following features from [1]. They show in particular that such sublevel sets are in general nothing but complements of suitable open balls.

Proposition 3 Let $x^{\star} \in X, \alpha \in \mathbb{R}$ and $\left.r \in\right] 0,+\infty[$. One has

$$
Q_{x^{\star}, r, \alpha}= \begin{cases}X \backslash B\left(r x^{\star}, \sqrt{r^{2}\left\|x^{\star}\right\|^{2}-2 r \alpha}\right) & \text { if } r^{2}\left\|x^{\star}\right\|^{2}-2 r \alpha>0, \\ X & \text { otherwise. }\end{cases}
$$

In particular, if $\varphi_{S, r}\left(x^{\star}\right)=\alpha \in \mathbb{R}$ for some closed subset $S$ of $X$, one has

$$
r^{2}\left\|x^{\star}\right\|^{2}-2 r \alpha=d_{S}^{2}\left(r x^{\star}\right)
$$

and

$$
Q_{x^{\star}, r, \alpha}= \begin{cases}X \backslash B\left(r x^{\star}, d_{S}\left(r x^{\star}\right)\right) & \text { if } r x^{\star} \notin S, \\ X & \text { otherwise. }\end{cases}
$$

With $\rho:=r^{2}\left\|x^{\star}\right\|^{2}-2 r \alpha$, one has

$$
\operatorname{bdry} Q_{x^{\star}, r, \alpha}=\left\{x \in X:\left\langle x^{\star}, x\right\rangle-\frac{\|x\|^{2}}{2 r}=\alpha\right\}=S\left(r x^{\star}, \sqrt{\rho}\right) .
$$

Further, for every $x \in X \backslash Q_{x^{\star}, r, \alpha}$, one has

$$
\operatorname{Proj}_{Q_{x^{\star}, r, \alpha}}(x)= \begin{cases}S\left(r x^{\star}, \sqrt{\rho}\right) & \text { if } x=r x^{\star}, \\ \left\{\frac{\sqrt{\rho}}{\left\|x-r x^{\star}\right\|}\left(x-r x^{\star}\right)+r x^{\star}\right\} & \text { otherwise. }\end{cases}
$$

In particular, the set $Q_{x^{\star}, r, \alpha}$ is proximinal and for every $x \in X$,

$$
d\left(x, Q_{x^{\star}, r, \alpha}\right)=\left(\sqrt{\rho}-\left\|x-r x^{\star}\right\|\right)^{+} .
$$

Coming back to the usual support function, it is worth recalling that a classical application of Hahn-Banach theorem says that for two closed convex sets $C_{1}, C_{2} \subset X$, one has

$$
\sigma\left(\cdot, C_{1}\right) \leq \sigma\left(\cdot, C_{2}\right) \Leftrightarrow C_{1} \subset C_{2}
$$


Our goal is now to show that $\varphi_{S, r}(\cdot)$ is a suitable function to extend such an equivalence to the $r$-prox-regular framework. First, it should be noted that for two nonempty closed subsets $S_{1}, S_{2}$ of $X$ and any real $r>0$,

$$
\varphi_{S_{1}, r}(\cdot) \leq \varphi_{S_{2}, r}(\cdot) \Leftrightarrow d_{S_{2}}(\cdot) \leq d_{S_{1}}(\cdot) \Leftrightarrow S_{1} \subset S_{2}
$$

Then, it turns out that the desired generalization reduces to the following question: under prox-regularity assumptions on $S_{1}$ and $S_{2}$, is it possible to weaken the inequality of the left hand-side of (20) in shrinking the set on which it is required? The answer is essentially positive and is contained in the next theorem. The following proposition which complements [10, Lemma 3.3] and [9, Theorem 6.10] is needed. Given an $r$-prox-regular subset of $X$ for some real $r>0$, recall that (see (19))

$\Lambda_{r}(S):=r \Gamma_{S, r}\left(\operatorname{Tube}_{r}(S)\right)=\left\{\left(1-\frac{r}{d_{S}(x)}\right) \operatorname{proj}_{S}(x)+\frac{r}{d_{S}(x)} x: x \in \operatorname{Tube}_{r}(S)\right\}$.

Proposition 4 Let $S$ be a nonempty subset of a real normed space $\left(E,\|\cdot\|_{E}\right)$ and let $r \in] 0,+\infty]$.

(a) One always has

$$
\operatorname{cl}_{E}\left(U_{r}(S)\right)=\operatorname{Enl}_{r}(S),
$$

or equivalently

$$
\operatorname{int}_{E}\left(\operatorname{Exte}_{r} S\right)=\left\{u \in E: d_{S}(u)>r\right\} .
$$

If $\left(E,\|\cdot\|_{E}\right)$ is the Hilbert space $(X,\|\cdot\|)$ and if $S$ is $r$-prox-regular, then the following assertions $(b)$ and $(c)$ hold.

(b) For every $s \in] 0, r[$, one has

$$
\operatorname{cl}_{X}\left(X \backslash \operatorname{Enl}_{s} S\right)=\left\{u \in X: d_{S}(u) \geq s\right\}=\operatorname{Ext}_{s}(S)
$$

or equivalently

$$
\operatorname{int}_{X}\left(\operatorname{Enl}_{s} S\right)=\left\{u \in X: d_{S}(u)<s\right\}=U_{s}(S)
$$

(c) One always has

$$
\Lambda_{r}(S)=\left\{x \in D_{r}(S): \operatorname{Proj}_{S}(x) \neq \emptyset\right\}
$$

along with

$$
D_{r}(S)=\operatorname{cl}_{X}\left(\Lambda_{r}(S)\right) \quad \text { and } \quad \operatorname{cl}_{w}\left(D_{r}(S)\right)=\operatorname{cl}_{w}\left(\Lambda_{r}(S)\right)
$$

Proof (a) We keep the notation $d_{S}$ (or $d(\cdot, S)$ ) for the distance function relative to the normed space $\left(E,\|\cdot\|_{E}\right)$. Fix any $\left.\left.r \in\right] 0,+\infty\right]$. Assume that $r<+\infty$, otherwise there is nothing to prove. The inclusion $\operatorname{cl}_{E} U_{r}(S) \subset \operatorname{Enl}_{r}(S)$ follows from the continuity of $d_{S}(\cdot)$. Let us establish the converse inclusion. Let $u \in$ $\operatorname{Enl}_{r}(S)$. We may suppose that $d_{S}(u)=r$. Let an arbitrary real $\varepsilon>0$. Pick any sequence $\left(z_{n}\right)_{n \in \mathbb{N}}$ of $S$ such that $r_{n}:=\left\|u-z_{n}\right\|_{E} \rightarrow r$. Choose any $N \in \mathbb{N}$ 
such that $r_{N}-r<\varepsilon$ and fix any $t \in[0,1]$ such that $1-\frac{r}{r_{N}}<t<\frac{\varepsilon}{r_{N}}$. Observe that $\left\|(1-t) u+t z_{N}-u\right\|_{E}=t r_{N}<\varepsilon$ and

$$
d\left((1-t) u+t z_{N}, S\right) \leq\left\|(1-t) u+t z_{N}-z_{N}\right\|_{E} \leq(1-t) r_{N}<r .
$$

Consequently, we have $(1-t) u+t z_{N} \in B(u, \varepsilon) \cap U_{r}(S) \neq \emptyset$. This means that $u \in \mathrm{cl}_{E} U_{r}(S)$. The desired equality in (a) follows.

Assume now that $\left(E,\|\cdot\|_{E}\right)$ is the Hilbert space $(X,\|\cdot\|)$ and that $S$ is $s$-proxregular.

(b) First, note that we always have $U_{s}(S) \subset \operatorname{int}_{X}\left(\operatorname{Enl}_{s}(S)\right)$, or equivalently

$$
\operatorname{cl}_{X}\left(X \backslash \operatorname{Enl}_{s} S\right) \subset X \backslash U_{s}(S)=\operatorname{Ext}_{s}(S) .
$$

Let us establish the converse inclusion. Fix any $u \in X \backslash U_{s}(S)$. We may suppose that $d_{S}(u)=s$. Let $\left(s_{n}\right)_{n \in \mathbb{N}}$ be a sequence of $] s, r\left[\right.$ with $s_{n} \rightarrow s$. Since $S$ is $r$-prox-regular, the set $\operatorname{Proj}_{S}(u)$ is reduced to a singleton (see Theorem 1(c)). Let $p \in X$ be such that $\operatorname{Proj}_{S}(u)=\{p\}$. Set for each $n \in \mathbb{N}$,

$$
u_{n}=p+s_{n} \frac{u-p}{\|u-p\|} .
$$

According to the $r$-prox-regularity of $S$, to the inclusion $u \in U_{r}(S) \backslash S$ and to the definition of $p$, we have by Theorem 1

$$
\{p\}=\operatorname{Proj}_{S}(u)=\operatorname{Proj}_{S}\left(u_{n}\right) \quad \text { for all } n \in \mathbb{N} \text {. }
$$

We also see that $d_{S}\left(u_{n}\right)=s_{n}>s$, so $u_{n} \in X \backslash \operatorname{Enl}_{s} S$ for each $n \in \mathbb{N}$. Further, $\left(u_{n}\right)_{n \in \mathbb{N}}$ converges to $p+s \frac{u-p}{\|u-p\|}$. Since $s=d_{S}(u)=\|u-p\|$, we have

$$
p+s \frac{u-p}{\|u-p\|}=u
$$

Consequently, we get $u \in \mathrm{cl}_{X}\left(X \backslash \operatorname{Enl}_{s} S\right)$.

(c) Set $x^{\star}:=\Gamma_{S, r}(x)$ with $x \in \operatorname{Tube}_{r}(S)$. First, observe that (18) and (19) entail

$$
\operatorname{proj}_{S}(x) \in \operatorname{Proj}_{S}\left(r x^{\star}\right) \text { and } r x^{\star} \in D_{r}(S) .
$$

From this we then note that the inclusion

$$
\Lambda_{r}(S) \subset\left\{u \in D_{r}(S): \operatorname{Proj}_{S}(u) \neq \emptyset\right\}
$$

holds true. Let us establish the converse inclusion. Fix any $u \in D_{r}(S)$ with $\operatorname{Proj}_{S}(u) \neq \emptyset$. Choose any $p \in \operatorname{Proj}_{S}(u) \neq \emptyset$ and $\left.x \in\right] p, u[$. Let us start by observing that

$$
p=\operatorname{proj}_{S}(x) \quad \text { and } \quad d_{S}(u)=r=d_{S}(x)+\|x-u\|,
$$


so in particular $x \in \operatorname{Tube}_{r}(S)$. With $d:=d_{S}(x)$, it follows

$$
\begin{aligned}
\left\|\left(1-\frac{r}{d}\right) p+\frac{r}{d} x-u\right\|^{2}= & \left\|\left(1-\frac{r}{d}\right)(p-u)+\frac{r}{d}(x-u)\right\|^{2} \\
= & \left(\frac{r}{d}-1\right)^{2}\|p-u\|^{2}+\frac{r^{2}}{d^{2}}\|x-u\|^{2} \\
& +2\left(1-\frac{r}{d}\right) \frac{r}{d}\langle p-u, x-u\rangle .
\end{aligned}
$$

On the other hand (keep in mind the inclusion $x \in] p, u[$ )

$$
\langle p-u, x-u\rangle=\|p-u\|\|x-u\|=r(r-d) .
$$

Combining the two latter equalities together gives

$$
\left\|\left(1-\frac{r}{d}\right) p+\frac{r}{d} x-u\right\|^{2}=\frac{2 r^{2}}{d^{2}}(r-d)^{2}-\frac{2 r^{2}}{d^{2}}(r-d)^{2}=0 .
$$

This entails that $u=\left(1-\frac{r}{d}\right) p+\frac{r}{d} x$, hence $u \in \Lambda_{r}(S)$ according to the inclusion $x \in \operatorname{Tube}_{r}(S)$ and to the equality $p=\operatorname{proj}_{S}(x)$. The first equality of $(c)$ is then established.

Let us show the second equality claimed by $(c)$. Let $x \in D_{r}(S)$. According to $(a)$ above, we can choose a sequence $\left(x_{n}\right)_{n \geq 1}$ in $\operatorname{Tube}_{r}(S)$ such that $x_{n} \rightarrow$ $x$. For every integer $n \geq 1$, set $x_{n}^{\star}:=\Gamma_{S, r}\left(x_{n}\right)$. From the boundedness of $\left(\operatorname{proj}_{S}\left(x_{n}\right)\right)_{n \geq 1}$ and the convergence $\frac{1}{r}-\frac{1}{d_{S}\left(x_{n}\right)} \rightarrow 0$, we observe that $x_{n}^{\star} \rightarrow \frac{x}{r}$, or equivalently $r x_{n}^{\star} \rightarrow x$. On the other hand, from (23) we also see that $\left(r x_{n}^{\star}\right)_{n \geq 1}$ is a sequence of $\Lambda_{r}(S)$. It follows that $D_{r}(S) \subset \operatorname{cl}_{\|\cdot\|}\left(\Lambda_{r}(S)\right)$. The converse inclusion follows from (22) proved above.

It remains to show the third equality in $(c)$. Since $\mathrm{cl}_{\|\cdot\|}\left(\Lambda_{r}(S)\right) \subset \mathrm{cl}_{w}\left(\Lambda_{r}(S)\right)$, from the previous equality we deduce that $D_{r}(S) \subset \operatorname{cl}_{w}\left(\Lambda_{r}(S)\right)$. It results that $\operatorname{cl}_{w}\left(D_{r}(S)\right) \subset \operatorname{cl}_{w}\left(\Lambda_{r}(S)\right)$, which is in fact an equality thanks to (22). The proof is complete.

Remark 2 If $s \in\{0, r\}$, then (21) does not hold in general. Indeed, in the case $s=0,(21)$ means $\operatorname{int}_{X}(S)=\emptyset$. Now, let us focus on the case $s=r$. Consider the set $S=\{t \in \mathbb{R}:|t| \geq 1\}$ which is $r$-prox-regular with $r:=1$. It is readily seen that $\operatorname{Enl}_{r}(S)=\mathbb{R}$ and $U_{r}(S)=\mathbb{R} \backslash\{0\}$, hence

$$
\emptyset=\operatorname{cl}_{\mathbb{R}}\left(\mathbb{R} \backslash \operatorname{Enl}_{r}(S)\right) \neq \operatorname{Ext}_{r}(S)=\{0\} .
$$

Now, we can state and prove the result on the consequence of the inequality $\varphi_{S_{1}, r}(\cdot) \leq \varphi_{S_{2}, r}(\cdot)$ on suitable subsets of $X$. Recall first that for a nonempty subset $A$ of a topological space $(T, \tau), A$ is $\tau$-connected if and only if for each $\tau$-closed subsets $F, G$ of $X$ satisfying $A \subset F \cup G$ and $A \cap F \cap G=\emptyset$, one has

$$
A \cap F=\emptyset \quad \text { or } \quad A \cap G=\emptyset .
$$


Theorem 4 Let $S_{1}, S_{2}$ be two $r$-prox-regular subsets of $X$ with $\left.\left.r \in\right] 0,+\infty\right]$. Then, one has

$$
S_{1} \subset S_{2} \cup \operatorname{Exte}_{r}\left(S_{2}\right)
$$

under anyone of the following conditions:

(a) $\varphi_{S_{1}, r}\left(x^{\star}\right) \leq \varphi_{S_{2}, r}\left(x^{\star}\right)$ for every $x^{\star} \in \Gamma_{S_{2}, r}\left(\operatorname{Tube}_{r}\left(S_{2}\right)\right)$;

(b) $r<+\infty$ and $\varphi_{S_{1}}\left(x^{\star}\right) \leq \varphi_{S_{2}}\left(x^{\star}\right)$ for every $x^{\star} \in \Lambda_{r}\left(S_{2}\right)$;

(c) $r<+\infty$ and $\varphi_{S_{1}}\left(x^{\star}\right) \leq \varphi_{S_{2}}\left(x^{\star}\right)$ for every $x^{\star} \in D_{r}\left(S_{2}\right)$.

If in addition $S_{1}$ is connected (which is always the case if diam $S_{1}<2 r$ ), then one has

$$
\begin{cases}S_{1} \subset S_{2} & \text { if } S_{1} \cap S_{2} \neq \emptyset \\ S_{1} \subset \operatorname{Exte}_{r}\left(S_{2}\right) & \text { otherwise }\end{cases}
$$

whenever one of the conditions $(a)-(c)$ holds.

Proof We may assume that $r<+\infty$. We observe through the equality valid for any subset $S \subset X$

$$
r \varphi_{S, r}\left(x^{\star}\right)=\varphi_{S}\left(r x^{\star}\right) \quad \text { for all } x^{\star} \in X
$$

that $(a) \Leftrightarrow(b)$. On the other hand, through the equality $D_{r}\left(S_{2}\right)=\operatorname{cl}_{\|\cdot\|}\left(\Lambda_{r}\left(S_{2}\right)\right)$ due to Proposition 4(c) and through the continuity of $\varphi_{S_{2}}(\cdot)$, we also see that the assertion $(b)$ is equivalent to $(c)$. As a consequence, it suffices to establish the desired result under the assumption $(a)$. Assume that $\varphi_{S_{1}, r}(\cdot) \leq \varphi_{S_{2}, r}(\cdot)$ on $\Gamma_{S_{2}, r}\left(\right.$ Tube $\left._{r}\left(S_{2}\right)\right)$. By contradiction, suppose that there is $a \in S_{1}$ with $a \notin S_{2} \cup \operatorname{Exte}_{r}\left(S_{2}\right)$, i.e., $a \in U_{r}\left(S_{2}\right) \backslash S_{2}=\operatorname{Tube}_{r}\left(S_{2}\right)$. According to Theorem 3 , there are $x^{\star} \in \Gamma_{S_{2}, r}\left(\operatorname{Tube}_{r}\left(S_{2}\right)\right)$ and a real $\alpha$ such that

$$
S_{2} \subset\left\{\left\langle x^{\star}, \cdot\right\rangle-\frac{\|\cdot\|^{2}}{2 r} \leq \alpha\right\} \quad \text { and } \quad\left\langle x^{\star}, a\right\rangle-\frac{\|a\|^{2}}{2 r}>\alpha .
$$

Hence, we have for all $x \in S_{2}$,

$$
\left\langle x^{\star}, x\right\rangle-\frac{\|x\|^{2}}{2 r} \leq \alpha<\left\langle x^{\star}, a\right\rangle-\frac{\|a\|^{2}}{2 r} \leq \varphi_{S_{1}, r}\left(x^{\star}\right),
$$

and this entails $\varphi_{S_{2}, r}\left(x^{\star}\right)<\varphi_{S_{1}, r}\left(x^{\star}\right)$ which is the desired contradiction. If in addition $S_{1}$ is connected, it suffices to note that $F:=S_{2}$ and $G:=$ $\operatorname{Exte}_{r}\left(S_{2}\right)$ are closed subsets of $X$ satisfying

$$
S_{1} \subset F \cup G \text { and } \quad S_{1} \cap F \cap G=\emptyset
$$

and to use the equivalence (24) recalled above.

Corollary 1 Let $S_{1}, S_{2}$ be two $r$-prox-regular connected subsets of $X$ for some $r \in] 0,+\infty]$ with $S_{1} \cap S_{2} \neq \emptyset$. Then, one has

$$
S_{1}=S_{2} \Leftrightarrow \varphi_{S_{1}, r}\left(x^{\star}\right)=\varphi_{S_{2}, r}\left(x^{\star}\right) \quad \text { for all } x^{\star} \in \bigcup_{i=1}^{2} \Gamma_{S_{i}, r}\left(\operatorname{Tube}_{r}\left(S_{i}\right)\right) .
$$

If $r<+\infty$, then one has

$$
S_{1}=S_{2} \Leftrightarrow \varphi_{S_{1}}\left(x^{\star}\right)=\varphi_{S_{2}}\left(x^{\star}\right) \quad \text { for all } x^{\star} \in D_{r}\left(S_{1}\right) \cup D_{r}\left(S_{2}\right) \text {. }
$$


Given a nonempty closed convex subset $C$ of $X$, it is well-known that

$$
C=\bigcap_{x^{\star} \in X}\left\{x \in X:\left\langle x^{\star}, x\right\rangle \leq \sigma\left(x^{\star}, C\right)\right\},
$$

that is, $C$ is the intersection of the closed half-spaces which contain it. It is worth pointing out that the latter equality can be written as

$$
C=\bigcap_{x^{\star} \in \Gamma_{C, \infty}\left(\operatorname{Tube}_{\infty}(C)\right)}\left\{x \in X:\left\langle x^{\star}, x\right\rangle \leq \sigma\left(x^{\star}, C\right)\right\},
$$

where $\Gamma_{C, \infty}\left(\operatorname{Tube}_{\infty}(C)\right)=\left\{\frac{u-\operatorname{proj}_{C}(u)}{d_{C}(u)}: u \in X \backslash C\right\}$. Then, it is quite natural to investigate if (25) could be extended to the context of an $r$-prox-regular set $S$ with the help of the function $\varphi_{S, r}$ and the set $\Gamma_{S, r}\left(\operatorname{Tube}_{r}(S)\right)$.

Let us start with the following lemma which will play a great role in some places in the rest of the paper.

Lemma 1 Let $\left.x^{\star} \in X, \alpha \in \mathbb{R}, r \in\right] 0,+\infty\left[\right.$. Assume that $S \subset Q_{x^{\star}, r, \alpha}$ and $x \notin Q_{x^{\star}, r, \alpha}$. Then, one has $\beta:=\varphi_{S, r}\left(x^{\star}\right) \leq \alpha$ and

$$
S \subset Q_{x^{\star}, r, \beta} \quad \text { and } \quad x \notin Q_{x^{\star}, r, \beta} .
$$

Further, one has

$$
d\left(x, Q_{x^{\star}, r, \alpha}\right) \leq d\left(x, Q_{x^{\star}, r, \beta}\right) \leq d(x, S) .
$$

Proof Set $Q:=Q_{x^{\star}, r, \alpha}$ and $Q^{\prime}:=Q_{x^{\star}, r, \beta}$. From the definition of $\beta$ and $Q_{x^{\star}, r, \beta}$ one sees that $S \subset Q^{\prime}$, then $d\left(x, Q^{\prime}\right) \leq d(x, S)$. Further, note that from the inclusion $S \subset Q$, we must have $\beta \leq \alpha$, then $Q^{\prime} \subset Q$ which in turn ensures that $d(x, Q) \leq d\left(x, Q^{\prime}\right)$ and $x \notin Q^{\prime}$ since $x \notin Q$.

Given a nonempty closed subset of $X$ and any real $r>0$, we have with $\mathcal{Q}_{r}:=\left\{Q_{x^{\star}, r, \alpha}: x^{\star} \in X, \alpha \in \mathbb{R}\right\}$

$$
S=\bigcap_{S \subset Q \in \mathcal{Q}_{r}} Q=\bigcap_{x^{\star} \in X} Q_{x^{\star}, r, \varphi_{S, r}\left(x^{\star}\right)}=\bigcap_{r x^{\star} \notin S} X \backslash B\left(r x^{\star}, d_{S}\left(r x^{\star}\right)\right)=: \mathcal{I} .
$$

Indeed, it directly follows from Lemma 1 and Proposition 3 that the three intersections involved above are equals. This along with the obvious inclusion $S \subset \bigcap_{S \subset Q \in \mathcal{Q}_{r}} Q$ justify that we only need to establish that $\mathcal{I} \subset S$. By contradiction, if there is $x \in \mathcal{I}$ such that $x \notin S$, we must have $x \notin B\left(u, d_{S}(u)\right)$ for every $u \in X \backslash S$, in particular $x \notin B\left(x, d_{S}(x)\right)$ and this cannot hold true.

Taking into account what precedes and (25) leads to consider the intersection

$\bigcap_{x^{\star} \in \Gamma_{S, r}\left(\operatorname{Tube}_{r}(S)\right)}\left\{x \in X: q_{x^{\star}, r}(x) \leq \varphi_{S, r}\left(x^{*}\right)\right\}=\bigcap_{x^{\star} \in \Gamma_{S, r}\left(\operatorname{Tube}_{r}(S)\right)} Q_{x^{\star}, r, \varphi_{S, r}\left(x^{*}\right)}$. 
Theorem 5 Let $S$ be an $r$-prox-regular set of $X$ for some real $r>0$. Then, one has

$$
S \cup \operatorname{Exte}_{2 r}(S)=\bigcap_{x^{\star} \in \Gamma_{S, r}\left(\operatorname{Tube}_{r}(S)\right)} Q_{x^{\star}, r, \varphi_{S, r}\left(x^{\star}\right)},
$$

or equivalently

$$
\operatorname{Tube}_{2 r}(S)=\bigcup_{x^{\star} \in \Lambda_{r}(S)} B\left(x^{\star}, r\right) .
$$

In particular, with $\Omega_{r}(S):=\Lambda_{r}(S) \cup\left\{x \in X: d_{S}(x) \geq 2 r\right\}$, one has the following equality

$$
X \backslash S=\bigcup_{x^{\star} \in \Omega_{r}(S)} B\left(x^{\star}, r\right) .
$$

Proof First, note that the equivalence claimed comes from the fact that by Proposition 3 and (19)

$$
\begin{aligned}
X \backslash \bigcup_{x^{\star} \in \Lambda_{r}(S)} B\left(x^{\star}, r\right) & =\bigcap_{x^{\star} \in \Gamma_{S, r}\left(\operatorname{Tube}_{r}(S)\right)} X \backslash B\left(r x^{\star}, d_{S}\left(r x^{\star}\right)\right) \\
& =\bigcap_{x^{\star} \in \Gamma_{S, r}\left(\operatorname{Tube}_{r}(S)\right)} Q_{x^{\star}, r, \varphi_{S, r}\left(x^{\star}\right)} .
\end{aligned}
$$

Fix any $x_{0} \in \bigcup_{x^{\star} \in \Lambda_{r}(S)} B\left(x^{\star}, r\right)$. There is $u \in \operatorname{Tube}_{r}(S)$ with $\left\|u^{\star}-x_{0}\right\|<r$, where $u^{\star}:=r \Gamma_{S, r}(u)$. Since

$$
\left\|u^{\star}-\operatorname{proj}_{S}(u)\right\|=\left\|r\left(\frac{1}{r}-\frac{1}{d_{S}(u)}\right) \operatorname{proj}_{S}(u)+\frac{r}{d_{S}(u)} u-\operatorname{proj}_{S}(u)\right\|=r
$$

we have

$$
d_{S}\left(x_{0}\right) \leq\left\|x_{0}-\operatorname{proj}_{S}(u)\right\| \leq\left\|x_{0}-u^{*}\right\|+\left\|u^{*}-\operatorname{proj}_{S}(u)\right\|<2 r .
$$

Thus, we get

$$
\bigcup_{x^{\star} \in \Lambda_{r}(S)} B\left(x^{\star}, r\right) \subset \operatorname{Tube}_{2 r}(S) .
$$

Now, fix any $\bar{x} \in X \backslash\left(S \cup \operatorname{Exte}_{r}(S)\right)=\operatorname{Tube}_{r}(S)$. From Theorem 3, we know that there exist $u^{\star} \in \Gamma_{S, r}\left(\operatorname{Tube}_{r}(S)\right)$ and $\alpha \in \mathbb{R}$ such that $S \subset Q_{u^{\star}, r, \alpha}$ and $\bar{x} \notin Q_{u^{\star}, r, \alpha}$. According to Lemma 1, we have $S \subset Q_{u^{\star}, r, \beta}$ and $x \notin Q_{u^{\star}, r, \beta}$, where $\beta:=\sup _{y \in S}\left(\left\langle u^{\star}, y\right\rangle-\frac{\left\|u^{\star}\right\|^{2}}{2 r}\right)=\varphi_{S, r}\left(u^{\star}\right) \leq \alpha<+\infty$. Hence, we get

$$
\bar{x} \notin \bigcap_{x^{\star} \in \Gamma_{S, r}\left(\operatorname{Tube}_{r}(S)\right)} Q_{x^{\star}, r, \varphi_{S, r}\left(x^{\star}\right)},
$$

and this says that

$$
\bigcap_{x^{\star} \in \Gamma_{S, r}\left(\operatorname{Tube}_{r}(S)\right)} Q_{x^{\star}, r, \varphi_{S, r}\left(x^{\star}\right)} \subset S \cup \operatorname{Exte}_{r}(S) .
$$


Taking complements in (28) and (27), and then using(29), we arrive to

$$
\operatorname{Tube}_{r}(S) \subset \bigcup_{x^{\star} \in \Lambda_{r}(S)} B\left(x^{\star}, r\right) \subset \operatorname{Tube}_{2 r}(S) .
$$

Now, let $x \in \operatorname{Tube}_{2 r}(S) \backslash \operatorname{Tube}_{r}(S)$. According to [9, Lemma 6.3], we have

$$
d:=d\left(x, D_{r}(S)\right)=d(x, S)-r<2 r-r=r .
$$

Fix any real $\varepsilon>0$ such that $d+\varepsilon<r$. There is $u \in D_{r}(S)$ such that $d \leq\|x-u\|<d+\varepsilon / 2$. Thanks to the equality $D_{r}(S)=\operatorname{cl}_{\|\cdot\|}\left(\Lambda_{r}(S)\right)$ in Proposition 4(c), there is $x^{\star} \in \Lambda_{r}(S)$ such that $\left\|x^{\star}-u\right\|<\varepsilon / 2$. It follows that

$$
\left\|x-x^{\star}\right\| \leq\|x-u\|+\left\|x^{\star}-u\right\|<d+\varepsilon<r,
$$

i.e., $x \in B\left(x^{\star}, r\right)$. This justifies the equality

$$
\operatorname{Tube}_{2 r}(S)=\bigcup_{x^{\star} \in \Lambda_{r}(S)} B\left(x^{\star}, r\right)
$$

or equivalently

$$
S \cup \operatorname{Exte}_{2 r}(S)=\bigcap_{x^{\star} \in \Gamma_{S, r}\left(\operatorname{Tube}_{r}(S)\right)} Q_{x^{\star}, r, \varphi_{S, r}\left(x^{\star}\right)} .
$$

The proof is complete.

Remark 3 We derive from the latter result that the set $\bigcap_{x^{\star} \in \Gamma_{S, r}\left(\operatorname{Tube}_{r}(S)\right)} Q_{x^{\star}, r, \varphi_{S, r}\left(x^{\star}\right)}$ fails to be connected if $\operatorname{Exte}_{2 r}(S) \neq \emptyset$.

\section{Distance function from a prox-regular set}

Our aim in the present section is to provide several new links between $d_{S}(\cdot)$ and $\varphi_{S, r}(\cdot)$ for an $r$-prox-regular set. Doing so, we will extend in particular two among the most basic results of convex analysis.

The first one (which is sometimes called analytic formulation of distance from a convex set, see, e.g., [20, Theorem 6.23]) asserts that for any nonempty closed convex subset $C$ of $X$ and for any $x \in X \backslash C$, there is one and only one $x^{\star} \in \mathbb{S}\left(\right.$ namely, $\left.x^{\star}=\Gamma_{C, \infty}(x)=d_{C}(x)^{-1}\left(x-P_{C}(x)\right)\right)$ such that

$$
d_{C}(x)=\left\langle x^{\star}, x\right\rangle-\sigma\left(x^{\star}, C\right) .
$$

The extension to the prox-regular setting is stated as follows.

Theorem 6 Let $S$ be an r-prox-regular subset of $X$ for some $r \in] 0,+\infty]$ and let $x \in \operatorname{Tube}_{r}(S)$. Then, there exists one and only one $x^{\star} \in X$ with $\left\|x^{\star}-r^{-1} x\right\|=1-r^{-1} d_{S}(x)$ (namely, $x^{\star}:=\Gamma_{S, r}(x)$ ) such that

$$
d_{S}(x)\left(1-(2 r)^{-1} d_{S}(x)\right)=q_{x^{\star}, r}(x)-\varphi_{S, r}\left(x^{\star}\right) .
$$

In particular, one has $\varphi_{S, r}\left(x^{\star}\right)<q_{x^{\star}, r}(x)$. 
Proof Existence. Set $p:=\operatorname{proj}_{S}(x)$ and $d:=d_{S}(x)$. Since $x-p \in \mathrm{N}^{P}(S ; p)$ (see (4)) we have (thanks to Theorem 1)

$$
\langle x-p, y-p\rangle \leq(2 r)^{-1} d\|y-p\|^{2} \quad \text { for all } y \in S,
$$

which gives $d^{2}=\inf _{y \in S}\left(\langle x-p, x-y\rangle+(2 r)^{-1} d\|y-p\|^{2}\right)$, or equivalently

$$
d=\inf _{y \in S}\left(\left\langle d^{-1}(x-p), x-y\right\rangle+(2 r)^{-1}\|y-p\|^{2}\right) .
$$

From the latter equality we also see that

$$
d-(2 r)^{-1} d^{2}=\inf _{y \in S}\left(\left\langle d^{-1}(x-p), x-y\right\rangle+(2 r)^{-1}\left(\|y-p\|^{2}-\|x-p\|^{2}\right)\right) .
$$

Put $g(y):=\left\langle d^{-1}(x-p), x-y\right\rangle+(2 r)^{-1}\left(\|y-p\|^{2}-\|x-p\|^{2}\right)$ for all $y \in S$ and $x^{*}:=d^{-1}(x-p)+r^{-1} p$. Note that

$$
g(y)=\left\langle x^{*}, x\right\rangle-(2 r)^{-1}\|x\|^{2}-\left\langle x^{*}, y\right\rangle+(2 r)^{-1}\|y\|^{2} \quad \text { for all } y \in S .
$$

The desired equality $d\left(1-(2 r)^{-1} d\right)=q_{x^{*}, r}(x)-\varphi_{S, r}\left(x^{*}\right)$ then follows.

Uniqueness. Let $x_{1}^{\star}, x_{2}^{\star} \in X$ such that for each $i \in\{1,2\}$

$$
\left\|x_{i}^{\star}-r^{-1} x\right\|=1-r^{-1} d \quad \text { and } \quad d\left(1-(2 r)^{-1} d\right)=q_{i}(x)-\varphi_{S, r}\left(x_{i}^{\star}\right),
$$

with $q_{i}:=q_{x_{i}^{\star}, r}$. Set $x^{\star}:=2^{-1}\left(x_{1}^{\star}+x_{2}^{\star}\right)$ and observe that

$$
\left\|x^{\star}-r^{-1} x\right\| \leq 2^{-1}\left\|x_{1}^{\star}-r^{-1} x\right\|+2^{-1}\left\|x_{2}^{\star}-r^{-1} x\right\|=1-r^{-1} d .
$$

Thanks to the definition of $x^{*}$ we notice that

$$
\begin{aligned}
q_{x^{\star}, r}(x)=2^{-1}\left[\left\langle x_{1}^{\star}+x_{2}^{\star}, x\right\rangle-\frac{1}{r}\|x\|^{2}\right] & =2^{-1}\left[\left\langle x_{1}^{\star}, x\right\rangle-\frac{1}{2 r}\|x\|^{2}+\left\langle x_{2}^{\star}, x\right\rangle-\frac{1}{2 r}\|x\|^{2}\right] \\
& =2^{-1}\left[q_{1}(x)+q_{2}(x)\right],
\end{aligned}
$$

which in turn ensures (see (15)) that

$$
\varphi_{S, r}\left(x^{\star}\right)=\sup _{u \in S} q_{x^{\star}, r}(u)=2^{-1} \sup _{u \in S}\left[q_{1}(u)+q_{2}(u)\right] .
$$

It results that

$$
\begin{aligned}
q_{x^{\star}, r}(x)-\varphi_{S, r}\left(x^{\star}\right) & =2^{-1}\left[q_{1}(x)+q_{2}(x)-\sup _{u \in S}\left(q_{1}(u)+q_{2}(u)\right)\right] \\
& \geq 2^{-1}\left[q_{1}(x)+q_{2}(x)-\sup _{u \in S} q_{1}(u)-\sup _{u \in S} q_{2}(u)\right] \\
& =2^{-1}\left(q_{1}(x)-\varphi_{S, r}\left(x_{1}^{\star}\right)+q_{2}(x)-\varphi_{S, r}\left(x_{2}^{\star}\right)\right) \\
& =d\left(1-(2 r)^{-1} d\right)>0,
\end{aligned}
$$

where the latter equality is due to the choice of $x_{1}^{\star}$ and $x_{2}^{\star}$. From the inequality $q_{x^{\star}, r}(x)>\varphi_{S, r}\left(x^{\star}\right)$, it is readily seen that $x \notin Q_{x^{\star}, r, \varphi_{S, r}\left(x^{\star}\right)}$. According to Proposition 3 , we see that $\zeta:=d^{2}\left(x, Q_{x^{\star}, r, \varphi_{S, r}\left(x^{\star}\right)}\right)=\left(\sqrt{\rho}-\left\|x-r x^{\star}\right\|\right)^{2}$, with 
$\rho:=r^{2}\left\|x^{\star}\right\|^{2}-2 r \varphi_{S, r}\left(x^{\star}\right)$. Elementary computations along with the equality $\rho=d_{S}^{2}\left(r x^{\star}\right)$ (see Proposition 1(a)) yield

$$
\begin{aligned}
\zeta & =2\left(r^{2}\left\|x^{\star}\right\|^{2}-r \varphi_{S, r}\left(x^{\star}\right)+2^{-1}\|x\|^{2}-r\left\langle x^{\star}, x\right\rangle-r d_{S}\left(r x^{\star}\right)\left\|x^{\star}-r^{-1} x\right\|\right) \\
& =2\left(r\left\langle x^{\star}, x\right\rangle-\|x\|^{2} / 2-r \varphi_{S, r}\left(x^{\star}\right)+r^{2}\left\|x^{\star}\right\|^{2}-2 r\left\langle x^{\star}, x\right\rangle+\|x\|^{2}-r d_{S}\left(r x^{\star}\right)\left\|x^{\star}-r^{-1} x\right\|\right) \\
& =2 r\left(q_{x^{\star}, r}(x)-\varphi_{S, r}\left(x^{\star}\right)\right)+2\left\|r x^{\star}-x\right\|^{2}-2 r d_{S}\left(r x^{\star}\right)\left\|x^{\star}-r^{-1} x\right\| .
\end{aligned}
$$

Consequently, we obtain

$$
q_{x^{\star}, r}(x)-\varphi_{S, r}\left(x^{\star}\right)=(2 r)^{-1} \zeta+\left\|x^{\star}-r^{-1} x\right\|\left(d_{S}\left(r x^{\star}\right)-r\left\|x^{\star}-r^{-1} x\right\|\right) .
$$

Putting together the latter inequality, the inclusion $S \subset Q_{x^{\star}, r, \varphi_{S, r}\left(x^{\star}\right)}$, the inequality $d_{S}\left(r x^{\star}\right) \leq d+\left\|r x^{\star}-x\right\|$ and (31) and (32), we arrive to

$$
\begin{aligned}
q_{x^{\star}, r}(x)-\varphi_{S, r}\left(x^{\star}\right) & \leq(2 r)^{-1} d^{2}+\left\|x^{\star}-r^{-1} x\right\| d \\
& \leq(2 r)^{-1} d^{2}+\left(1-r^{-1} d\right) d \\
& =d\left(1-(2 r)^{-1} d\right) \leq q_{x^{\star}, r}(x)-\varphi_{S, r}\left(x^{\star}\right) .
\end{aligned}
$$

Then, it follows that $\left\|x^{\star}-r^{-1} x\right\|=1-r^{-1} d=: \alpha$, in particular the three points $x_{1}^{\star}, x_{2}^{\star}, x^{\star}$ lie on the sphere $r^{-1} x+\alpha \mathbb{S}_{X}$ with $x^{\star}=\left(x_{1}^{\star}+x_{2}^{\star}\right) / 2$. The strict convexity of this sphere of the Hilbert space $X$ ensures that $x_{1}^{\star}=x_{2}^{\star}$, which justifies the uniqueness property.

Given a (nonempty closed) convex set $C$, a direct computation gives for every $y \in C$ and every $u^{\star} \in X$ with $\left\|u^{\star}\right\|=1$,

$$
\left\langle u^{\star}, x\right\rangle-\sigma\left(u^{\star}, C\right)=\inf _{y \in C}\left\langle u^{\star}, x-y\right\rangle \leq d_{C}(x) .
$$

Hence, taking into account (30), we have

$$
d_{C}(x)=\max _{\left\|x^{\star}\right\|=1}\left(\left\langle x^{\star}, x\right\rangle-\sigma\left(x^{\star}, C\right)\right)=\max _{\left\|x^{\star}\right\|=1} \inf _{y \in C}\left\langle x^{\star}, x-y\right\rangle .
$$

Let us point out that we can reverse maximum/supremum and infimum in the latter formula (see, e.g., [20, Remarks 7.2,p.126]), i.e.,

$$
d_{C}(x)=\max _{x^{\star} \in \mathbb{S}} \inf _{y \in C}\left\langle x^{\star}, x-y\right\rangle=\inf _{y \in C} \max _{x^{\star} \in \mathbb{S}}\left\langle x^{\star}, x-y\right\rangle .
$$

The next result provides a similar feature for prox-regular sets.

Proposition 5 Let $S$ be an $r$-prox-regular subset of $X$ with $r \in] 0,+\infty], x \in$ $\operatorname{Tube}_{r}(S)$ and $\mathcal{L}:=\left\{x^{\star} \in X:\left\|x^{\star}-r^{-1} x\right\|=1-r^{-1} d_{S}(x)\right\}$. Then, one has

$d_{S}(x)\left(1-\frac{d_{S}(x)}{2 r}\right)=\max _{x^{\star} \in \mathcal{L}} \inf _{y \in S}\left(q_{x^{\star}, r}(x)-q_{x^{\star}, r}(y)\right)=\inf _{y \in S} \max _{x^{\star} \in \mathcal{L}}\left(q_{x^{\star}, r}(x)-q_{x^{\star}, r}(y)\right)$. 
Proof Let us focus on the first equality. Fix any $v^{\star} \in \mathcal{L}$ and observe that

$$
\begin{aligned}
\inf _{y \in S}\left(\frac{\|y\|^{2}-\|x\|^{2}}{2 r}+\left\langle v^{\star}, x-y\right\rangle\right) & =\inf _{y \in S}\left(\left\langle v^{\star}-\frac{x}{r}, x-y\right\rangle+\frac{\|x-y\|^{2}}{2 r}\right) \\
& \leq \inf _{y \in S}\left(\left\|v^{\star}-\frac{x}{r}\right\|\|x-y\|+\frac{\|x-y\|^{2}}{2 r}\right) \\
& =\kappa,
\end{aligned}
$$

with $\kappa:=\inf _{y \in S}\left(\left(1-\frac{d_{S}(x)}{r}\right)\|x-y\|+\frac{\|x-y\|^{2}}{2 r}\right)$. By contradiction, suppose that $\kappa>\left(1-\frac{d_{S}(x)}{r}\right) d_{S}(x)+\frac{d_{S}^{2}(x)}{2 r}=: \theta$. Let $\left.\varepsilon \in\right] \theta, \kappa\left[\right.$. Pick any sequence $\left(y_{n}\right)_{n \in \mathbb{N}}$ of $S$ with $\left\|x-y_{n}\right\| \rightarrow d_{S}(x)$ and note that for every $n \in \mathbb{N}$,

$$
\left(1-\frac{d_{S}(x)}{r}\right)\left\|x-y_{n}\right\|+\frac{\left\|x-y_{n}\right\|^{2}}{2 r} \geq \kappa>\varepsilon>\theta .
$$

Passing to the limit then gives $\left(1-\frac{d_{S}(x)}{r}\right) d_{S}(x)+\frac{1}{2 r} d_{S}^{2}(x) \geq \varepsilon>\theta$ and this cannot hold true. Hence, we have

$$
\inf _{y \in S}\left(\frac{\|y\|^{2}-\|x\|^{2}}{2 r}+\left\langle v^{\star}, x-y\right\rangle\right) \leq \kappa \leq \theta=d_{S}(x)\left(1-\frac{d_{S}(x)}{2 r}\right),
$$

where the first inequality is due to (33). Taking the supremum yields

$$
d_{S}(x)\left(1-\frac{d_{S}(x)}{2 r}\right) \geq \sup _{x^{\star} \in \mathcal{L}} \inf _{y \in S}\left(\frac{\|y\|^{2}-\|x\|^{2}}{2 r}+\left\langle x^{\star}, x-y\right\rangle\right) .
$$

On other hand, using Theorem 6 , there is $u^{\star} \in \mathcal{L}$ such that

$$
\begin{aligned}
d_{S}(x)\left(1-\frac{d_{S}(x)}{2 r}\right) & =q_{u^{\star}, r}(x)-\sup q_{u^{\star}, r}(S) \\
& =\inf _{y \in S}\left(\frac{\|y\|^{2}-\|x\|^{2}}{2 r}+\left\langle u^{\star}, x-y\right\rangle\right) .
\end{aligned}
$$

It remains to combine what precedes with the latter inequality to get the first equality in the proposition. Now, let us establish the second equality of the proposition. Setting $\rho:=1-r^{-1} d_{S}(x)$ and writing

$$
\begin{aligned}
\sup _{x^{\star} \in \mathcal{L}} \frac{\|y\|^{2}-\|x\|^{2}}{2 r}+\left\langle x^{\star}, x-y\right\rangle & =\frac{\|y\|^{2}-\|x\|^{2}}{2 r}+\left\langle\frac{x}{r}, x-y\right\rangle+\sup _{\left\|z^{\star}\right\|=\rho}\left\langle z^{\star}, x-y\right\rangle \\
& =\frac{\|x-y\|^{2}}{2 r}+\rho \max _{\left\|z^{\star}\right\|=1}\left\langle z^{\star}, x-y\right\rangle \\
& =\frac{\|x-y\|^{2}}{2 r}+\rho\|x-y\|,
\end{aligned}
$$


we see that

$$
\begin{aligned}
\sup _{x^{\star} \in \mathcal{L}} \frac{\|y\|^{2}-\|x\|^{2}}{2 r}+\left\langle x^{\star}, x-y\right\rangle & =\max _{x^{\star} \in \mathcal{L}} \frac{\|y\|^{2}-\|x\|^{2}}{2 r}+\left\langle x^{\star}, x-y\right\rangle \\
& =\frac{\|x-y\|^{2}}{2 r}+\rho\|x-y\| .
\end{aligned}
$$

Consequently, we arrive to

$$
\inf _{y \in S} \max _{x^{\star} \in \mathcal{L}} \frac{\|y\|^{2}-\|x\|^{2}}{2 r}+\left\langle x^{\star}, x-y\right\rangle=\inf _{y \in S} \frac{\|x-y\|^{2}}{2 r}+\rho\|x-y\|=\kappa,
$$

which completes the proof.

Remark 4 A function $f: C \rightarrow \mathbb{R} \cup\{+\infty\}$ on a convex subset $C$ of $X$ is recalled to be $\sigma$-semiconvex (on $C$ ) for some $\sigma \in \mathbb{R}_{+}:=[0,+\infty[$ if (see, e.g., [34])

$$
f(t x+(1-t) y) \leq t f(x)+(1-t) f(y)+\frac{\sigma}{2} t(1-t)\|x-y\|^{2},
$$

for all $x, y \in C$ and for all $t \in] 0,1[$. Recall also (see, e.g., $[6,27,34])$ that the closed set $S$ in $X$ is $r$-prox-regular for some real $r>0$ if and only if the function $d_{S}(\cdot)$ is $(r-s)^{-1}$-semiconvex on any convex subset $C \subset U_{s}(S)$ with $s<r$, or equivalently $d_{S}+\psi_{C}$ is $(r-s)^{-1}$-semiconvex on $X$. Assuming the $r$-prox-regularity of the set $S$ and using the above semiconvexity property, it is derived in [1] from properties of the Legendre-Fenchel $r$-conjugate that, for any nonempty closed convex $C \subset U_{s}(S)$

$$
\left(d_{S}+\psi_{C}\right)(x)=\sup _{x^{\star} \in X}\left(q_{x^{\star}, r-s}(x)-\left(d_{S}+\psi_{C}\right)^{\star, r-s}\left(x^{\star}\right)\right) \quad \text { for all } x \in X,
$$

which can be rewritten as

$d_{S}(x)=\sup _{x^{\star} \in X} \inf _{y \in C}\left(\left\langle x^{\star}, x-y\right\rangle+\frac{1}{2(r-s)}\left(\|y\|^{2}-\|x\|^{2}\right)+d_{S}(y)\right) \quad$ for all $x \in C$.

The second crucial result coming from convex analysis that we will extend can be seen as a geometrical counterpart of (30). Indeed, given a nonempty closed convex subset $C$ of $X$ and $x \in X \backslash C$, it is known (see, e.g., [20, Theorem 6.25]) that the distance $d(x, C)$ of $x$ from $C$ coincides with the maximum of $d(x, H)$ taken over all hyperplanes $H$ which separate $C$ and $x$, and this maximum is attained for one and only one such hyperplane.

Theorem 7 Let $S$ be an $r$-prox-regular subset of $X$ for some $r \in] 0,+\infty[$, and $x \in \operatorname{Tube}_{r}(S)$. Then, one has

$$
d(x, S)=\max \left\{d\left(x, Q_{y^{\star}, r, \alpha}\right):\left(y^{\star}, \alpha\right) \in X \times \mathbb{R}, S \subset Q_{y^{\star}, r, \alpha}, x \notin Q_{y^{\star}, r, \alpha}\right\} .
$$


The maximum is attained at $\left(x^{\star}, \varphi_{S, r}\left(x^{\star}\right)\right)$ with $x^{\star}:=\Gamma_{S, r}(x)$ and

$$
\operatorname{proj}_{S}(x)=\operatorname{proj}_{Q_{x^{\star}, r, \varphi_{S, r}\left(x^{\star}\right)}}(x) .
$$

Further, for all $y^{\star} \in X$ with $\left\|y^{\star}-r^{-1} x\right\|=1-r^{-1} d_{S}(x)$ and all $\alpha \in \mathbb{R}$, one has the following implication

$$
\left.\begin{array}{l}
d(x, S)=d\left(x, Q_{y^{\star}, r, \alpha}\right), \\
S \subset Q_{y^{\star}, r, \alpha}, x \notin Q_{y^{\star}, r, \alpha}
\end{array}\right\} \Rightarrow\left(y^{\star}, \alpha\right)=\left(x^{\star}, \varphi_{S, r}\left(x^{\star}\right)\right) .
$$

Proof Set $x^{\star}:=\Gamma_{S, r}(x), d:=d_{S}(x), p:=\operatorname{proj}_{S}(x)$ and $\beta:=\varphi_{S, r}\left(x^{\star}\right)$. We have already established (see Lemma 1$)$

$$
\sup \left\{d\left(x, Q_{y^{\star}, r, \alpha}\right):\left(y^{\star}, \alpha\right) \in X \times \mathbb{R}, S \subset Q_{y^{\star}, r, \alpha}, x \notin Q_{y^{\star}, r, \alpha}\right\} \leq d .
$$

By Theorem 3, there is $t \in \mathbb{R}$ such that $S \subset Q_{x^{\star}, r, t}$ and $x \notin Q_{x^{\star}, r, t}$. Thanks to Lemma 1, we have $S \subset Q_{x^{\star}, r, \beta}$ and $x \notin Q_{x^{\star}, r, \beta}$. On the other hand, from Theorem 6, we get $\beta=\varphi_{S, r}\left(x^{\star}\right)=\left\langle x^{\star}, x\right\rangle-\frac{\|x\|^{2}}{2 r}-d\left(1-(2 r)^{-1} d\right)$. It is routine to check that $r^{2}\left\|x^{\star}\right\|^{2}=\left\|r x^{\star}-x\right\|^{2}+\|x\|^{2}+2\left\langle r x^{\star}-x, x\right\rangle$ and

$$
-2 r \beta=-2 r\left\langle x^{\star}, x\right\rangle+2 r d\left(1-(2 r)^{-1} d\right)+\|x\|^{2} .
$$

Putting the two latter equalities together yields

$$
r^{2}\left\|x^{\star}\right\|^{2}-2 r \beta=\left\|r x^{\star}-x\right\|^{2}+2 r d\left(1-(2 r)^{-1} d\right) .
$$

It is also readily seen that $r x^{\star}-x=\left(1-r d^{-1}\right)(p-x)$, so

$$
\left\|r x^{\star}-x\right\|^{2}+2 r d\left(1-(2 r)^{-1} d\right)=\left(1-r d^{-1}\right)^{2} d^{2}+2 r d\left(1-(2 r)^{-1} d\right)=r^{2} .
$$

Using Proposition 3 and the equality (34), we obtain

$$
d\left(x, Q_{x^{\star}, r, \beta}\right)=\sqrt{\left\|r x^{\star}-x\right\|^{2}+2 r d\left(1-(2 r)^{-1} d\right)}-\left\|r x^{\star}-x\right\| .
$$

Putting this, (35) and the inclusion $x \in \operatorname{Tube}_{r}(S)$ together we arrive to

$$
d\left(x, Q_{x^{\star}, r, \beta}\right)=r-\left|1-d^{-1} r\right| d=d .
$$

Now, fix any $y^{\star} \in X$ with $\left\|y^{\star}-\frac{x}{r}\right\|=1-r^{-1} d$ and $t \in \mathbb{R}$. Assume that $d(x, S)=d\left(x, Q_{y^{\star}, r, t}\right)$ along with $x \notin Q_{y^{\star}, r, t}$ and $S \subset Q_{y^{\star}, r, t}$. By virtue of Lemma 1, we have

$$
\theta:=\varphi_{S, r}\left(y^{\star}\right) \leq t \quad \text { and } \quad x \notin Q_{y^{\star}, r, \theta}
$$

and $d(x, S)=d\left(x, Q_{y^{\star}, r, t}\right) \leq d\left(x, Q_{y^{\star}, r, \theta}\right) \leq d(x, S)$. Via Proposition 3, we get

$$
d\left(x, Q_{y^{\star}, r, \theta}\right)=\sqrt{\rho}-\left\|x-r y^{\star}\right\| \quad \text { with } \quad \rho:=r^{2}\left\|y^{\star}\right\|^{2}-2 r \theta .
$$

According to Proposition 3 again, we know that $\rho=d^{2}\left(r y^{\star}, S\right)$, so

$$
\begin{aligned}
d=d\left(x, Q_{y^{\star}, r, \theta}\right) & =d\left(r y^{\star}, S\right)-\left\|x-r y^{\star}\right\| \\
& \leq d+\left\|x-r y^{\star}\right\|-\left\|x-r y^{\star}\right\|=d .
\end{aligned}
$$


Consequently, we have $d\left(r y^{\star}, S\right)=d+\left\|x-r y^{\star}\right\|$, or equivalently

$$
\rho=r^{2}\left\|y^{\star}\right\|^{2}-2 r \theta=\left(d+\left\|x-r y^{\star}\right\|\right)^{2} .
$$

Expanding the right-hand side of the latter equality and coming back to the definition of $\theta$ yield

$$
-2 r \varphi_{S, r}\left(y^{\star}\right)=d^{2}+2 d\left\|x-r y^{\star}\right\|+\|x\|^{2}-2 r\left\langle y^{\star}, x\right\rangle,
$$

which can be rewritten as $q_{y^{\star}, r}(x)-\varphi_{S, r}\left(y^{\star}\right)=(2 r)^{-1} d\left(d+2\left\|x-r y^{\star}\right\|\right)$. Putting the latter equality and the equality $\left\|y^{\star}-r^{-1} x\right\|=1-r^{-1} d$ together ensure that $q_{y^{\star}, r}(x)-\varphi_{S, r}\left(y^{\star}\right)=d\left(1-(2 r)^{-1} d\right)$. The uniqueness property provided by Theorem 6 then guarantees that $x^{\star}=y^{\star}$. It remains to show that $t=\varphi_{S, r}\left(y^{\star}\right)$, or equivalently (see 36) $t \geq \varphi_{S, r}\left(y^{\star}\right)$. If $t<\varphi_{S, r}\left(y^{\star}\right)$, we have

$$
\begin{aligned}
d=d\left(x, Q_{y^{\star}, r, t}\right)=d\left(x, Q_{x^{\star}, r, t}\right) & =\sqrt{r\left\|x^{\star}\right\|^{2}-2 r t}-\left\|x-r x^{\star}\right\| \\
& >\sqrt{r\left\|x^{\star}\right\|^{2}-2 r \varphi_{S, r}\left(x^{\star}\right)}-\left\|x-r x^{\star}\right\| \\
& =d\left(x, Q_{x^{\star}, r, \varphi_{S, r}\left(x^{\star}\right)}\right)=d
\end{aligned}
$$

which is a contradiction. The proof is complete.

Acknowledgement. The second author has received funding from the European Union's Horizon 2020 Research and Innovation Programme under the Marie Sklodowska-Curie Grant Agreement No 823731 CONMECH.

\section{References}

1. S. Adly, F. Nacry, L. Thibault, Prox-regular sets and Legendre-Fenchel transform: separation properties, available soon on arXiv.org

2. S. Adly, F. Nacry, L. Thibault, Preservation of Prox-Regularity of Sets with Applications to Constrained Optimization, SIAM J. Optim. 26 (2016), 448-473.

3. S. Adly, F. Nacry, L. Thibault, Prox-regularity approach to generalized equations and image projection, ESAIM: COCV, 24, 677-708, 2018.

4. J.-P. Aubin, I. Ekeland, Applied Nonlinear Analysis, Pure and Applied Mathematics (New York). A Wiley-Interscience Publication. John Wiley \& Sons, Inc., New York, 1984.

5. E. Asplund, Čebyšev sets in Hilbert space, Trans. Amer. Math. Soc. 144 (1969), 235-240.

6. M.V. Balashov, Weak convexity of the distance function, J. Convex Anal, 20 (2013), 93-106.

7. M.V. Balashov, G.E. Ivanov, Weakly convex and proximally smooth sets in Banach spaces, Izv. Math. 73 (2009), 455-499.

8. H. Berens, Best approximation in Hilbert space, in Approximation Theory III (Proc. Conf., Univ. Texas, Austin, Tex., 1980) (E. W. Cheney, ed.), Academic Press, New York (1980), 1-20.

9. F. Bernard, L. Thibault, N. Zlateva Characterizations of prox-regular sets in uniformly convex Banach spaces, J. Convex Anal. 13 (2006), 525-559.

10. M. Bounkhel, L. Thibault, Nonconvex sweeping process and prox-regularity in Hilbert space, Journal of Nonlinear and Convex Analysis, 6, 359-374 (2005).

11. J.M. Borwein, J.D. Vanderwerff, Convex Functions: constructions, characterizations and counterexamples, Encyclopedia of Mathematics and its Applications, 109. Cambridge University Press, Cambridge, 2010. 
12. A. Canino, On p-convex sets and geodesics, J. Differential Equations 75 (1988), 118-157.

13. F.H. Clarke, R.J. Stern, P.R. Wolenski, Proximal smoothness and the lower-C ${ }^{2}$ property, J. Convex Anal (1995), 117-144.

14. F.H. Clarke, Optimization and Nonsmooth Analysis, Second Edition, Classics in Applied Mathematics, 5, Society for Industrial and Applied Mathematics (SIAM), Philadelphia, PA, 1990.

15. F. H. Clarke, R. J Stern and P. R. Wolenski, Proximal smoothness and the lower-C2 property, J. Convex Analysis, 2 (1995), 117-144.

16. G. Colombo, L. Thibault, Prox-regular sets and applications, Handbook of nonconvex analysis and applications 99-182 (2010), Int. Press, Somerville, MA.

17. H. Federer, Curvature measures, Trans. Amer. Math. Soc. 93 (1959), 418-491.

18. V. Klee, Convexity of Chebyshev sets, Math. Annalen 142, 292-304 (1961).

19. G. Godini, On a problem of H. Berens, Math. Annalen 263, 279-281 (1983).

20. F.R. Deutsch, Best Approximations in Inner Product Spaces, CMS Books in Mathematics/Ouvrages de Mathématiques de la SMC, 7, Springer-Verlag, New-York, 2001.

21. V.V. Goncharov, G. E. Ivanov, Strong and weak convexity of closed sets in a Hilbert space, Operations research, engineering, and cyber security, 259297, Springer Optim. Appl., 113, Springer, Cham, 2017.

22. J.-B. Hiriart-Urruty, Ensembles de Tchebychev vs ensembles convexes : l'état de la situation vu via l'analyse convexe non lisse, Ann. Sci. Math. Québec 22 (1998), 47-62.

23. J.-B. Hiriart-Urruty, La conjecture des points les plus éloignés revisités, Ann. Sci. Math. Québec 29 (2005), 197-214.

24. G.E. Ivanov, Weakly Convex Sets and Functions: Theory and Applications (Fizmatlit, Moscow, 2006) (in Russian).

25. A. Jourani, E. Vilches, Positively $\alpha$-Far Sets and Existence Results for Generalized Perturbed Sweeping Processes, J. Convex. Anal. 23 (2016), 775-821.

26. B.S. Mordukhovich, Variational Analysis and Generalized Differentiation I, Grundlehren Series Vol. 330, Springer, 2006.

27. F. Nacry, L. Thibault, Regularization of sweeping process: old and new, Pure Appl. Funct. Anal. 4 (2019), 59-117.

28. J.-P. Penot, Calculus Without Derivatives, Graduate Texts in Mathematics, 266. Springer, New-York, 2013.

29. R.A. Poliquin and R.T. Rockafellar, Prox-regular functions in variational analysis, Trans. Amer. math. Soc. 348 (1996), 18051838.

30. R.A. Poliquin, R.T. Rockafellar, L. Thibault, Local differentiability of distance functions, Trans. Amer. Math. Soc. 352 (2000), 5231-5249.

31. R.T. Rockafellar, R.J.-B. Wets, Variational Analysis, Grundlehren der Mathematischen Wissenschaften, vol. 317. Springer, New York, 1998.

32. A. Shapiro, Existence and differentiability of metric projections in Hilbert spaces, SIAM J. Optimization 4 (1994), 130-141.

33. L. Thibault, Subsmooth functions and sets, Linear Nonlinear Anal. 4 (2018), 157-269.

34. L. Thibault, Unilateral Variational Analysis in Banach Spaces, to appear.

35. J.-P. Vial, Strong and weak convexity of sets and functions, Math. Oper. Res. 8 (1983), 231-259. 